\title{
A MEASUREMENT-THEORETIC FOUNDATION OF RULE INTERESTINGNESS EVALUATION
}

\author{
A Thesis \\ Submitted to the Faculty of Graduate Studies and Research \\ In Partial Fulfillment of the Requirements \\ FOR THE DEGREE OF \\ MASTER OF ScIENCE \\ IN \\ COMPUTER SCIENCE \\ UNIVERSITY OF REGINA
}

By

Yaohua Chen

Regina, Saskatchewan

August 31, 2004

(C) Copyright 2004: Yaohua Chen 


$\begin{array}{ll}\begin{array}{l}\text { Library and } \\ \text { Archives Canada }\end{array} & \begin{array}{l}\text { Bibliothèque et } \\ \text { Archives Canada }\end{array} \\ \begin{array}{l}\text { Published Heritage } \\ \text { Branch }\end{array} & \begin{array}{l}\text { Direction du } \\ \text { Patrimoine de l'édition }\end{array} \\ \begin{array}{l}\text { 395 Wellington Street } \\ \text { Ottawa ON K1A ON4 }\end{array} & \begin{array}{l}\text { 395, rue Wellington } \\ \text { Ottawa ON K1A ON4 } \\ \text { Canada }\end{array} \\ \end{array}$

Your file Votre référence ISBN: 0-612-96085-4 Ourfile Notre référence ISBN: 0-612-96085-4

The author has granted a nonexclusive license allowing the Library and Archives Canada to reproduce, loan, distribute or sell copies of this thesis in microform, paper or electronic formats.

The author retains ownership of the copyright in this thesis. Neither the thesis nor substantial extracts from it may be printed or otherwise reproduced without the author's permission.
L'auteur a accordé une licence non exclusive permettant à la Bibliothèque et Archives Canada de reproduire, prêter, distribuer ou vendre des copies de cette thèse sous la forme de microfiche/film, de reproduction sur papier ou sur format électronique.

L'auteur conserve la propriété du droit d'auteur qui protège cette thèse. $\mathrm{Ni}$ la thèse ni des extraits substantiels de celle-ci ne doivent être imprimés ou aturement reproduits sans son autorisation.
In compliance with the Canadian Privacy Act some supporting forms may have been removed from this thesis.

While these forms may be included in the document page count, their removal does not represent any loss of content from the thesis.
Conformément à la loi canadienne sur la protection de la vie privée, quelques formulaires secondaires ont été enlevés de cette thèse.

Bien que ces formulaires aient inclus dans la pagination, il n'y aura aucun contenu manquant.

\section{Canadä}




\title{
UNIVERSITY OF REGINA
}

\section{FACULTY OF GRADUATE STUDIES AND RESEARCH}

\section{SUPERVISORY AND EXAMINING COMMITTEE}

\author{
Yaohua Chen, candidate for the degree of Master of Science, has presented a thesis titled, \\ A Measurement-Theoretic Foundation of Rule Interestingness Evaluation, in an oral \\ examination held on August 30, 2004. The following committee members have found \\ the thesis acceptable in form and content, and that the candidate demonstrated \\ satisfactory knowledge of the subject material. \\ External Examiner: $\quad$ Dr. Gang Zhao, Faculty of Engineering \\ Co-Supervisor: Dr. Yiyu Yao, Department of Computer Science \\ Co-Supervisor: Dr. Xue Dong Yang, Department of Computer Science \\ Committee Member: Dr. Robert Hilderman, Department of Computer Science
}

Chair of Defense: $\quad$ Dr. Dianliang Deng, Department of Mathematics \& Statistics 


\section{Abstract}

Knowledge Discovery in Databases (KDD) is the efficient extraction of implicitly hidden, previously unknown, and potentially useful information and knowledge from databases. One of the important tasks in KDD is rule interestingness evaluation in order to prune useless or non-interesting rules. Many measures have been proposed and studied extensively. They are normally defined by structural characteristics or statistical information about the rules. The meaningfulness of each measure is interpreted based on some intuitive argument or mathematical properties. Through reviewing existing studies of rule interestingness measures, the existing studies are classified in several ways, such as subjective measures, objective measures, and so on. Each type reflects certain characteristics of rules.

Based on the review of existing studies, it is found that a general and unified framework does not appear to exist. Furthermore, the user judgement is not explicitly corporate in rule interestingness evaluation. Therefore, it is necessary to build such

a framework in which one is able to represent explicitly, precisely, and formally the 
user preferences. Since usefulness of discovered rules must be eventually judged by users, such a framework is appealing. We show that measurement theory can be used to establish a solid foundation for rule interestingness evaluation. A framework is based on the notion of user preference. The objective of this thesis is to establish a measurement-theoretic foundation of rule interestingness evaluation, which is an important topic of the foundations of KDD. 


\section{Acknowledgments}

I would like to express many thanks to my co-supervisors Dr. Yiyu Yao and Dr. Xuedong Yang, who provided a motivating, enthusiastic, and critical research atmosphere, stimulated my interest in this special topic, critical advice and encouragement. It was a great pleasure to me to conduct this thesis under their supervision. I also would like to acknowledge with much appreciation the crucial role of my internal examiner, Dr. Robert J. Hilderman, for his valuable comments to my thesis.

I gratefully acknowledge the generous financial support of the Faculty of Graduate Studies and Research, Natural Science and Engineering Research Council of Canada (NSERC), and the Computer Science Department.

Especially, I would like to give my special thanks to my dear wife Linshu Wang, my parents, and friends for their constant love, encouragement and moral support. 


\section{Post Acknowledgments}

I also would like to give much appreciation to the external examiner, Dr. Gang Zhao, the Faculty of Engineering, for his valuable comments and special attention to my thesis. Thanks also to Dr. Dianliang Deng for chairing my thesis defence. 


\section{Contents}

Abstract $\quad$ i

Acknowledgments

Post Acknowledgments iv

Table of Contents $\quad$ v

List of Tables viii

List of Figures $\quad$ ix

Chapter 1 Introduction 1

1.1 Statement of the Problem . . . . . . . . . . . . . . 1

1.1.1 Rule Interestingness Evaluation . . . . . . . . . . . 4

1.1.2 Approach and Objective of Thesis . . . . . . . . . . 6

1.2 Contributions of Thesis . . . . . . . . . . . . . . 8

1.3 Organization of Thesis ................... 9

V 
2.1 Information Tables and (Discovered) Rules . . . . . . . . . . . . 12

2.2 Introduction to Evaluation . . . . . . . . . . . . . 15

2.2.1 Definition of Evaluation ............. 15

2.2.2 Procedure of Evaluation . . . . . . . . . . . 17

2.2.3 Measurement of Evaluation . . . . . . . . . . 18

2.2.4 Subjectivity of Evaluation . . . . . . . . . . 18

2.3 The Roles of Rule Interestingness Evaluation . . . . . . . . . . . . . . 19

2.4 A Review of Existing Studies _. . . . . . . . . . . . . 21

2.4.1 Subjective and Objective Measures . . . . . . . . . . 21

2.4.2 Association and Correlation Measures . . . . . . . . . 25

2.4.3 Deviation and Change Measures . . . . . . . . . . . 34

2.4.4 Single Rule and Multiple Rules . . . . . . . . . . . . 36

2.4.5 Axiomatic Approaches . . . . . . . . . . . . . 36

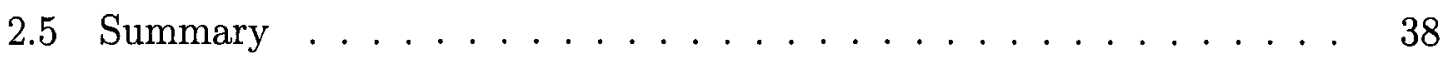

\section{$\begin{array}{lll}\text { Chapter } 3 & \text { Overview of Measurement Theory } & 39\end{array}$}

3.1 Basic Ideas of the Measurement Theory . . . . . . . . . . . . . . 39

3.2 Relational Systems . . . . . . . . . . . . . . . . 41

3.3 Axioms of the Empirical System . . . . . . . . . . . . . . . 42

3.4 Homomorphism of Relational Systems . . . . . . . . . . . . . . 43 
3.5 Procedure of Measurement . . . . . . . . . . . . . . . . 44

Chapter 4 Application of Measurement Theory to Rule Interest$\begin{array}{ll}\text { ingness Evaluation } & 46\end{array}$

4.1 User Preference Relations . . . . . . . . . . . . . . . . . . 47

4.2 Axioms of User Preference Relation . . . . . . . . . . . . . . . . . . 48

4.3 Homomorphism based on Real-valued Function . . . . . . . . . . . 50

4.4 Ordinal Measurement of Rules Interestingness . . . . . . . . . . . 51

4.5 An Inductive Learning Procedure for Linear Combination Measure . . 53

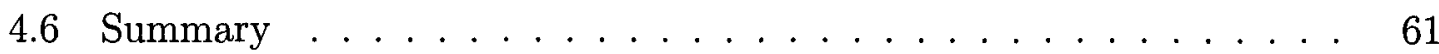

$\begin{array}{lll}\text { Chapter } 5 & \text { Conclusion and Future Research } & 62\end{array}$

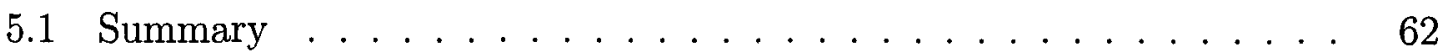

5.2 Future Research . . . . . . . . . . . . . . 64

vii 


\section{List of Tables}

2.1 An information table . . . . . . . . . . . . . . 13

2.2 Contingency table for concepts $\phi$ and $\psi \ldots \ldots$. . . . . . . . . . 26

2.3 Contingency table with cardinality . . . . . . . . . . . 26

2.4 An information table of sales force versus yearly sales revenue . . . . 30

2.5 An example of information table . . . . . . . . . . . . 33

2.6 Another example of information table . . . . . . . . . . . 33

2.7 A table of confidence distributions of association rules . . . . . . . . 35

viii 


\section{List of Figures}

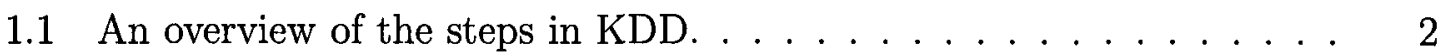

2.1 A diagram of correlation between two concepts $X$ and $Y \ldots \ldots 31$ 


\section{Chapter 1}

\section{Introduction}

\subsection{Statement of the Problem}

Knowledge Discovery in Databases (KDD) has emerged as a new area of research in computer science. The objective of KDD systems is to extract implicitly hidden, previously unknown, and potentially useful information and knowledge from databases [19]. It is generally accepted that KDD is an interactive and iterative process consisting of many phases [10, 16, 25, 44, 64].

Fayyad et al. suggested that the standard KDD process consists of five significant steps illustrated in Figure 1.1 [17]. Furthermore, they presented several more detailed phases, including developing and understanding of the application domain, creating a target data set, data cleaning, data reduction and projection, choosing the data mining task, choosing the data mining algorithm(s), data mining, interpreting mined patterns, and consolidating and acting on the discovered knowledge $[16,17]$. We 


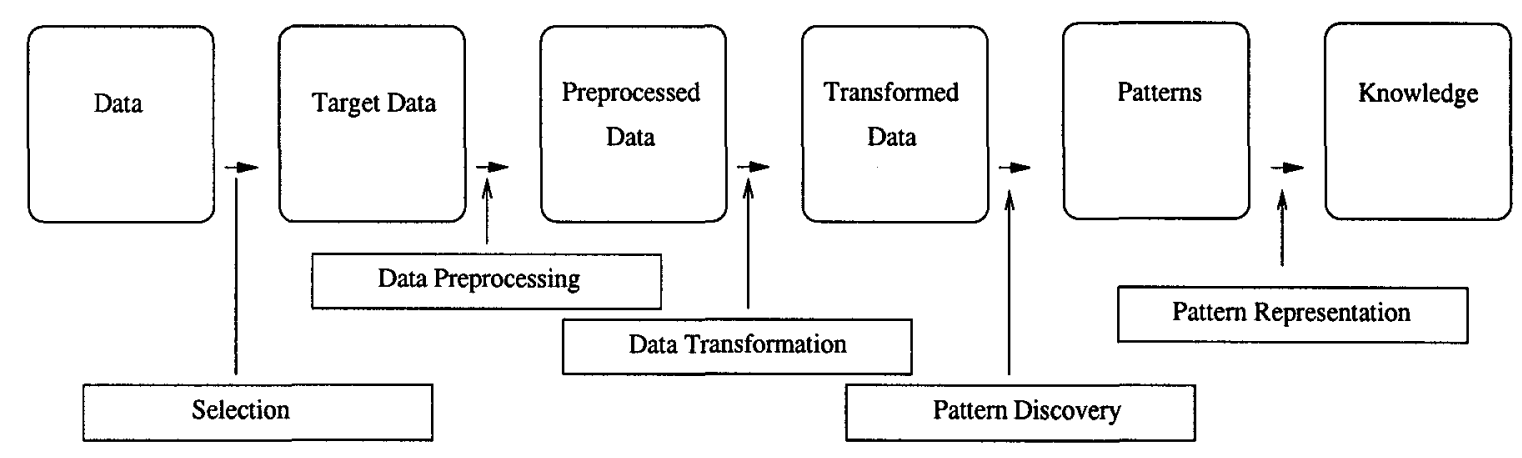

Figure 1.1: An overview of the steps in KDD.

summarize those phases into main five steps:

- Selection attempts to analyze the application domain and prepare original and raw data. Users determine what domain(s) of knowledge they want to find and collect an appropriate target data set. The phases involved in this step include developing and understanding of the application domain, data selection, and creating a target data set.

- Data Preprocessing attempts to perform data cleaning operations on the target data. Some operations, such as expanding abbreviations, covering missing data, and classifying number values of attributes, may be needed in this step.

- Data Transformation includes data reduction and projection to reduce the dimensions or size of data for pattern discovery step.

- Pattern Discovery can be simply called data mining, which chooses and performs algorithm(s) to extract the patterns from the target data set. As one of the 
central steps in KDD, it is the application of specific machine learning, statistics, and other data analysis techniques for knowledge extraction and abstraction. Fundamental tasks of the data mining step are the discovery and evaluation of patterns.

- Pattern Representation attempts to interpret, consolidate, and make applications of the discovered patterns.

Knowledge generated from a large data set or information table is often expressed in terms of a set of discovered rules or patterns [16]. There exist many kinds of expression methods or forms for discovered rules, such as association rules, classification rules, clusters, time series, sequential patterns, summaries, and others. Each may be viewed as a different type of knowledge. Furthermore, various types of knowledge are embedded in a large database [79].

The number of rules is typically huge and only a small portion of rules is actually useful [62]. An important problem in KDD is evaluation of interestingness of the mined rules and filtering out useless rules [62]. In this thesis, we concentrate on rule interestingness evaluation. 


\subsubsection{Rule Interestingness Evaluation}

Rule interestingness evaluation in KDD means rules as a type of knowledge are evaluated by users based on specific interestingness measures and criteria. For example, the evaluation of the degree of association (relationship) between items in a rule [1]. Given a large database of transactions, where each transaction consists of a set of items, associations between items can be found. An example of an association between items is the statement that $90 \%$ of transactions that purchase bread and butter also purchase milk. Such an association between items is expressed as an association rule. The number $90 \%$ is used to evaluate the rule's degree of association. If $90 \%$ is bigger than a user-defined threshold, then this association rule is recognized as an interesting rule.

Many measures have been proposed, such as conditional probability, correlation coefficient, distance metric, and others. Rule interestingness evaluation plays different important roles in different phases of the KDD process. For example, after a large number of discovered rules are generated from databases, a user may be required to evaluate each one to decide whether it is useful or interesting [27]. Nevertheless, in the data mining phase, quantitative measures can be used to reduce the size of search space [1].

As an important and crucial topic in KDD, rule interestingness evaluation has been given much attention. Many measures have been proposed and studied to quantify 
the usefulness or interestingness of rules $[20,26,61,62,87]$. Many studies investigate and compare rule interestingness measures based on some intuitive argument or some mathematical properties. For example, a summarized table proposed by Tan et al. is used to compare twenty-one measures for association rules [68]. Based on seven mathematical properties, they examine and discuss the measures and summarize the results in a table. Their discussion suggests that there is no measure that is better than others in all properties and application domains. Different measures have different essential properties. Their results lead to an in-depth understanding of different aspects of rules. In other words, the different measures reflect different application domains or user preferences where each measure reflects a certain characteristic of rules. For example, suppose we have discovered a rule of "if weather is sunshine and temperature is mild, then shopping is yes". This rule may be realized as an interesting rule by users because its probability of occurrence in a data set is more than a user-defined threshold. In this case, a rule's probability of occurrence is a certain feature of this rule and deemed as a rule interestingness measure. However, this rule may relatively be measured as a non-interesting rule because it has no surprisingness for users with respect to users' preferences.

Therefore, some fundamental problems in rule interestingness evaluation are: How do we examine or evaluate different types of interestingness measures? Is there a connection between users and measures? How can we represent the users in the 
measurement for rule interestingness evaluation?

In fact, one needs to link the meaningfulness of a measure to its usage or users. In theory, it may not be meaningful to argue which measure is better without reference to its roles and usage. There is a lack of a well-accepted framework of measurement for examining fundamental issues of rule interestingness evaluation in a systematic and unified manner. Furthermore, since usefulness or interestingness of mined rules must be eventually judged by users, consideration of user preference for the unified framework is necessary. A well-developed and unified framework should be able to represent the user preferences explicitly, precisely, and formally. In this thesis, we provide a review of existing studies and then present our work on a user-centered framework by using measurement theory.

\subsubsection{Approach and Objective of Thesis}

In this thesis, measurement theory is used to establish this framework of measurement for rule interestingness evaluation. The theory provides the necessary concepts and methodologies for the representation, classification, characterization, and interpretation of user preference. The basic idea of measurement theory is to choose and assign numbers to subjects when measuring subjects so that the numerical characteristics of the numbers can reflect the qualitative characteristics of the subjects. In other words, measurement theory is concerned with choosing consistent quantitative 
representations of qualitative subjects. The quantitative representations are called measures. In rule interestingness evaluation, the meaningfulness of a measure is determined by the user(s) perception of the interestingness or usefulness of rules.

The classification of the existing studies on rule interestingness evaluation can be performed in several ways. Each of them provides a different view. Most studies on rule interestingness evaluation concentrate on specific measures, and each measure reflects a certain aspect of rules. Existing studies of rule interestingness evaluation can be viewed as measure-centered approaches. Measures are used as primitive notions to quantify the interestingness of rules. Quantitative measures are typically interpreted using intuitively defined notions, such as novelty, usefulness, non-trivialness, and unexpectedness. The method that proposed in this thesis is a user-centered approach. The user can be a person, an organization, or a system. User judgment or preference, expressed by a binary relation on a set of rules, is used as a primitive notion to model rule interestingness. Measures are treated as a derived notion that provides a quantitative representation of user preference. For instance, a user preference may be defined by association of a rule based on an information table. In other words, a user will determine that one rule is more interesting than another rule if and only if the degree of association of the rule is greater than another rule's. Based on measurement theory, the association of a rule is used to define the user preference. The measures on the association are quantitative representations of the user preference. 
The study of rule interestingness evaluation needs to be connected to the study of foundations of KDD or data mining. Our recent paper also discusses this approach in detail [82].

\subsection{Contributions of Thesis}

The major contributions in this thesis are summarized as follows:

- We review existing studies for rule interestingness evaluation from several perspectives. We provide a more general review from several different points of views such as subjective measures, objective measures, roles of measures, and others. From the review, we found there is a need to establish a general and unified framework in which various rule interestingness evaluations can be formally and appropriately defined and interpreted.

- We establish a general and unified framework for measurement of rule interestingness. Measurement theory is used to build this general, unified, user-centered framework because of its necessary concepts and methodologies for the definition, classification, and characterization of user preference. The user judgments are expressed by user preferences. Our work can be viewed as an important topic in foundations of KDD or data mining. 
- We show that a combined measure can be inferred by inductive learning procedure from user preference on a training set of discovered rules. When evaluating the discovered rules, each measure reflects a particular perspective. A combined measure summarizes the information by several measures and may provide a better evaluation. We introduce a linear model of combined measure and then apply an inductive learning procedure for finding the coefficients of the linear function. It demonstrates that the proposed framework is very useful and flexible. One can easily apply results from measurement theory and related fields.

\subsection{Organization of Thesis}

In the next chapter, we give some background information about rules as knowledge and introduce basic notions and roles of rule interestingness evaluation. Then, we present a review of existing studies. Several classifications of the existing quantitative measures are presented based on application domains of measures. The review of existing studies suggests a general and unified framework to define, interpret, and compare different measures.

A brief overview of measurement theory is presented in Chapter 3. A concrete example is used to demonstrate basic notions of measurement theory. Then, we give the definitions of the basic concepts of measurement theory, such as relational system, 
axioms and homomorphism. Then fundamental steps of measurement are discussed in the same chapter.

Application of measurement theory to rule interestingness evaluation is demonstrated in the Chapter 4. Based on a definition of user preference, a framework of rule interestingness measurement is established using the methodologies of measurement theory. Some properties and an ordinal scale for the measurement of user preference of rules in this framework are also analyzed in this chapter. We introduce a linear model of combined measure and apply an inductive learning procedure for finding the coefficients of the linear measure.

Finally, we conclude our research and discuss future work in Chapter 5. 


\section{Chapter 2}

\section{Background and Related Work}

Knowledge generated from a large data set or information table is often expressed in terms of a set of discovered rules [16]. There exists many types of (discovered) rules and the number of rules is very large, but only a few of these rules are judged to be of some interest to the users. Rule interestingness evaluation plays a very important role in defining and filtering out useful or interesting rules.

This chapter is organized as follows. In Section 2.1, we introduce definitions of an information table and a (discovered) rule. In Section 2.2, we present an introduction of evaluation. In Section 2.3, we discuss the roles of rule interestingness evaluation for a KDD process and model. In Section 2.4, we propose a review of rule interestingness evaluation from various perspectives. 


\subsection{Information Tables and (Discovered) Rules}

An information table represents all available information. Knowledge or rules can be discovered based on the information table [49]. Yao introduced a formal model for KDD and data mining [81, 83]. Based on his model, we introduce definitions for information tables and (discovered) rules in this section.

The objects in an information table can be described by conjunctions of attributevalue pairs. The rows of an information table represent the objects. The columns describe a set of attributes. An example is illustrated in Table 2.1 where $o_{1}, o_{2}, \ldots$, $o_{10}$ are objects, "Weather", "Temperature", and "Shopping" are attributes, and each cell denotes a value of an object on an attribute. For instance, the value of object $o_{1}$ on attribute "Weather" is "Sunshine". An information table can be formally defined by a quadruple:

$$
S=\left(U, A t,\left\{V_{a} \mid a \in A t\right\},\left\{I_{a} \mid a \in A t\right\}\right)
$$

where $U$ is a finite nonempty set of objects, $A t$ is a finite nonempty set of attributes, $V_{a}$ is a nonempty set of values for $a \in A t$, and $I_{a}$ is an function to map from $U$ to $V_{a}$, that is, $I_{a}: U \rightarrow V_{a}$ for $a \in A t$.

With respect to the definition of information table given above, a language can be defined to express various types of rules [81]. In this language, an atomic formula is a pair $(a, v)$, where $a \in A t$ and $v \in V_{a}$. If $\phi$ and $\psi$ are fomulas, then so are $\neg \phi, \phi \wedge \psi, \phi \vee \psi, \phi \rightarrow \psi$, and $\phi \equiv \psi$ by applying the logic connectives $\neg, \wedge, \vee, \rightarrow$, 
Table 2.1: An information table

\begin{tabular}{|c|c|c|c|}
\hline Object & Weather & Temperature & Shopping \\
\hline$o_{1}$ & Sunshine & Mild & No \\
$o_{2}$ & Sunshine & Mild & Yes \\
$o_{3}$ & Rain & Cold & No \\
$o_{4}$ & Snow & Cold & No \\
$o_{5}$ & Sunshine & Mild & Yes \\
$o_{6}$ & Rain & Mild & Yes \\
$o_{7}$ & Sunshine & Mild & Yes \\
$o_{8}$ & Snow & Cold & No \\
$o_{9}$ & Sunshine & Mild & Yes \\
$o_{10}$ & Sunshine & Mild & Yes \\
\hline
\end{tabular}

and $\leftrightarrow$. For an atomic formula $\phi=(a, v)$, it is assumed that an object $x$ satisfies $\phi$ or does not satisfy $\phi$. In other words, if an object $x$ has a value of $v$ on the attribute $a$, then we say that the object $x$ satisfies $\phi$. Otherwise, we say $x$ does not satisfy $\phi$. The set of objects that satisfy a formula $\phi$ is denoted as $m(\phi)$. Thus, if the atomic formula is the pair of $(a, v)$, then the set of objects is $m(a, v)=\left\{x \in U \mid I_{a}(x)=v\right\}$. Then the following properties hold:

$$
\begin{aligned}
\text { (i) } & m(\neg \phi)=\neg m(\phi), \\
\text { (ii) } & m(\phi \wedge \psi)=m(\phi) \cap m(\psi), \\
\text { (iii) } & m(\phi \vee \psi)=m(\phi) \cup m(\psi), \\
\text { (iv) } & m(\phi \rightarrow \psi)=\neg m(\phi) \cup m(\psi), \\
\text { (v) } & m(\phi \equiv \psi)=(m(\phi) \cap m(\psi)) \cup(\neg m(\phi) \cap \neg m(\psi)) .
\end{aligned}
$$


The formula $\phi$ can be viewed as the description of a set of object $m(\phi)$.

In the study of formal concepts, every concept consists of two parts, the intention and extension [72, 73]. The set of objects is referred to as the extension, and the set of attributes as the intention of a concept. Therefore, a formula $\phi$ represents the intention of a concept and a subset of objects $m(\phi)$ denotes the extension of the concept. The pair $(\phi, m(\phi))$ is denoted as a concept. The concept $(\phi, m(\phi))$ can be described as the set of objects $m(\phi)$ that have the property expressed by the formula $\phi$. An example concept in Table 2.1 is given as follows. For the attribute $a=$ "Weather" and the value $v=$ "Sunshine", the formula $\phi$ is ("Weather", "Sunshine"). There are objects $m(\phi)=\left\{o_{1}, o_{2}, o_{5}, o_{7}, o_{9}, o_{10}\right\}$ satisfying or supporting $\phi$. The pair (("Weather", "Sunshine"), $\left.\left\{o_{1}, o_{2}, o_{5}, o_{7}, o_{9}, o_{10}\right\}\right)$ is a concept. The intention $\phi=$ ("Weather", "Sunshine") is comprehension of the concept, and the extension $m(\phi)=\left\{o_{1}, o_{2}, o_{5}, o_{7}, o_{9}, o_{10}\right\}$ includes instances belonging to the concept.

One of the important tasks of data mining or KDD is to find strong relationships between concepts in an information table [87]. A rule can be defined and represented as $\phi \Rightarrow \psi$, where $\phi$ and $\psi$ are intentions of two concepts [81]. The symbol $\Rightarrow$ in a rule $\phi \Rightarrow \psi$ represents simply a connection or relationship between two concepts $\phi$ and $\psi$. The meanings and interpretations of $\Rightarrow$ are varied based on users or application domains. Rules can be classified according to the interpretations of $\Rightarrow$. In other words, different interpretations of $\Rightarrow$ in a rule represent different types of knowledge. 
For example, an association rule $\phi \Rightarrow \psi$ describes an association relationship between two concepts $\phi$ and $\psi$. However, a classification rule $\phi \Rightarrow \psi$ presents a decision relationship between the two concepts. Furthermore, based on the extensions $m(\phi)$, $m(\psi)$, and $m(\phi \wedge \psi)$, various quantitative measures can be used for rule interestingness evaluation. A systematic analysis of quantitative measures associated with rules is given by Yao and Zhong [87].

\subsection{Introduction to Evaluation}

The discussion of the basic ideas of evaluation can improve our understanding of rule interestingness evaluation.

\subsubsection{Definition of Evaluation}

Many approaches define the term evaluation based on a specific view, such as qualitative assessment and detailed statistical analysis $[24,56,66]$. Suchman analyzed various definitions of evaluation with regard to particular uses of the term "evaluation", including both conceptual and operational approaches [66]. Generally, evaluation is determination of results attained by some activities for accomplishing some valued goals or objectives. Simply speaking, evaluation is a value judgment as to whether something is good or bad in some respects. The practice of evaluation can in fact be applied to many processes and research areas, such as reducing uncertainties, 
improving effectiveness, and making decisions [48].

Three basic components for an evaluation to occur are analyzed and studied by Geisler [24]. The first component is subjects for evaluation, which is what or who needs to be evaluated. For example, when evaluating the warmth of an object, the subject of this evaluation is warmth, a characteristic of the object. The formulation of the subjects is always done in the first step of an evaluation. More precisely distinguished subjects for an evaluation can produce a more exact framework, measurement, and result.

The users who are interested in and willing to perform the evaluation are considered as the second component of an evaluation. Knowing who will participate in or whom will benefit from the evaluation will help clarify why the evaluation is performed and which measures or methods should be used. Since the qualities of subjects must be eventually judged by users, an evaluation needs to consider the user judgment or user preference. The users can be the humans, organizations, or even systems. Different types of participants may have different purposes for conducting an evaluation and lead to different results.

The procedures for evaluation and concrete measures are an evaluation's third component. Clarification of the criteria for measurement and designing an implementation or procedure for an evaluation are the key points in this component. When one develops the procedures and measurements for an evaluation, she or he must consider 
the subjects and the users. As Suchman points out, the procedure of an evaluation can be constructed for different purpose, by different methods, with different criteria based on different users and subjects [66].

\subsubsection{Procedure of Evaluation}

According to the definition of evaluation, the procedure for an evaluation can be simply and generally described as follows $[24,66]$ :

- Identification of the subjects to be evaluated.

- Collection of data and information for the evaluation.

- Analysis of the data to measure subjects based on user preferences or criteria.

- Summarization of user judgments and conducting the process of an evaluation for decision making.

The real procedure of an evaluation can be very complicated and might be an iterative process [66]. Furthermore, identifying and accurately measuring or quantifying the subjects is very difficult to achieve. More often than not, an approximation approach can be accepted by general users. 


\subsubsection{Measurement of Evaluation}

During the procedure of an evaluation, measurement always plays a crucial role and measurement theory provides the necessary concepts and methodologies for the evaluation. The measurement can be generally described as estimating empirical objects or events, such as weight, color, or intelligence [50], and performed by assigning numbers to the objects or events in order that properties of objects or events can be represented as numerical properties [31]. In other words, the properties of the numbers are able to faithfully reflect the properties of objects or events. For example, we use degrees of temperature instead of simply distinguishing between warm objects and cold ones. The two commonly used scales of temperature, Celsius and Fahrenheit, are different measures on estimating the degree of temperature. In this thesis, we focus on the measurement of rule interestingness evaluation and provide a general and unified framework of measurement with respect to the user preference.

\subsubsection{Subjectivity of Evaluation}

Based on the definition and procedure of evaluation, Suchman argued that evaluation is an inherently subjective process [66]. The steps, methods, and measures used in an evaluation depend on users who participate in the evaluation. Selection of the criteria and measures reflects principles and underlying beliefs or interests of the users [24]. 
Mackie argued that subjective values are commonly used when one evaluates objects or events [42]. Objectivity is only connected to the objective measures and implementation of the measurement. People always judge the objects or events by their subjective interests. Different people have different judgments on the same thing because they always stand on their own interests or standards. In other words, a measurement is based on individual judgments, preferences or standards.

In fact, user preference is a very important issue that must be considered before an evaluation can occur [24]. It can be described as a user's discrimination on different subjects rationally [41]. The users can simply describe their preference as "they act upon their interests and desires they have" [15]. In measurement and decision theories, user preferences are used to present the user judgments or user interests and can be viewed as the standards of an evaluation $[21,41,50,57]$. Therefore, the user preference should be considered in the measurement of an evaluation.

\subsection{The Roles of Rule Interestingness Evaluation}

Rule interestingness evaluation plays at least three different roles in the KDD process. In the data mining phase, quantitative measures can be used to reduce the size of search space. An example is the use of the well known support measure, which reduces the number of item sets that need to be examined [1]. In the phase of interpreting mined patterns, rule interestingness evaluation plays a role in selecting 
the useful or interesting rules from the set of discovered rules [61,62]. For example, the confidence measure of association rules is used to select only strongly associated item sets [1]. Finally, in the phase of consolidating and acting on discovered knowledge, rule interestingness evaluation can be used to quantify the usefulness and effectiveness of discovered rules. For example, many measures such as cost, classification error, and classification accuracy for evaluating a classification rule play such a role [20].

Recently, Yao proposed a conceptual formulation of KDD in a three-layered framework consisting of a philosophy level, a technique level, and an application level [79]. The philosophy level focuses on formal characterization, description, representation, and classification of knowledge embedded in databases without reference to mining algorithms. It provides answers to the question: What is the knowledge embedded in databases? The technique level concentrates on data mining algorithms without reference to specific applications. It provides answers to the question: How to discover knowledge embedded in databases? The application level focuses on the use of discovered knowledge with respect to particular domains. It provides answers to the question: How to apply the discovered knowledge? With respect to the threelayered framework, the roles played by rule interestingness evaluation are described as follows. At the philosophy level, quantitative measures can be used to characterize and classify different types of rules. At the algorithm level, measures may be used to reduce the search space. At the application level, measures can be used to quantify 
the utility, profit, effectiveness, or actionability of discovered rules.

\subsection{A Review of Existing Studies}

Existing studies can be classified based on different perspectives. We summarize the main results from several points of views below.

\subsubsection{Subjective and Objective Measures}

Many measures can be classified into two categories consisting of objective measures and subjective measures [61]. Objective measures depend only on the structure of rules and the underlying data used in the discovery process. Subjective measures depend on the user who examines the rules $[35,61]$.

Measures defined by statistical and structural information are viewed as objective measures. They are application and domain independent. For example, Gago and Bento proposed a measure for selection of the discovered rules with the highest average distance between them [22]. The distance measure is developed based on the structural and statistical information of a rule such as the number of attributes in a rule and the values of attributes. A rule is deemed as interesting if it has the highest average distance to the others. Therefore, one does not need to consider the application and domain when measuring the discovered rules by using the distance measure. Information theoretic measures are also objective measures because they 
use the underlying data in a data set to evaluate the information content or entropy of a rule $[32,65,80,85,89]$.

A systematic analysis of objective measures is performed by using a $2 \times 2$ contingency table induced by a rule [87]. The results show that different classes of rules can be identified based on the objective measures, such as peculiarity rules (low support and high confidence), exception rules (low support and high confidence, but complement to other high support and high confidence rules), and outlier patterns (far away from the statistical mean) [88].

Although statistical and structural information provides an effective indicator of the potential effectiveness of a rule, its usefulness could be limited. One needs, then, to consider the subjective aspects of rules or explanations of rules [86]. Subjective measures consider the user who examines the rules. For example, Silberschatz and Tuzhilin proposed a subjective measure of rule interestingness based on the notion of unexpectedness and in terms of a user belief system [61, 62]. The basic idea of their measure is that discovered rules which have more unexpected information with comparison to a user belief system are deemed as more interesting. Thus, subjective measures are both application and user dependent. In other words, a user needs to incorporate other domain specific knowledge such as user interest, utility, value, profit, action-ability, etc. $[54,69]$. The interestingness or usefulness of rules are measured and interpreted based on domain specific notions. 
Many subjective measures are proposed. Profit or utility-based mining is a special kind of constraint-based mining, taking into account of both statistical significance and profit significance $[33,70]$. Doyle discussed the importance and usefulness of the notions of economic rationality and suggested that economic rationality can play a large role for measuring a rule [12]. The profit-based measures allow users to prune rules with high statistical significance, but generate low profit or high risk. Similarly, Barber and Hamilton proposed the notion of share measures which consider the contribution, in terms of profit, of an item in an item set [2].

Nevertheless, the measure in actionable rule mining deals with profit-driven actions required by business decision making $[34,38]$. A rule is referred to as actionable if the user can apply it to do something. An action may be designing some business strategies or promotions to change the non-desirable /non-profitable rules to desirable/profitable rules. Ras et al. introduced a notion of action rules [54, 55]. A special type of discovered rules can be constructed to present actions taken to improve profitability. For business users, actionable rule mining can help them to influence and control their changes or actions to obtain the highest profits from consumers. Ling et al. suggested that the economic profit notion can be used in measuring and mining optimal actions [34].

For example, a classification rule can distinguish which consumers are likely loyal or not loyal to an insurance company [34]. Based on this classification rule, it is very 
useful that the users can take some actions for improving or changing a consumers to loyalty. Similarly some association rules can be used to give a recommendation of products to consumers [70]. Different recommendation strategies will produce different profit margins and managers usually prefer plans to maximize profits.

Based on the notion of economic profit, we propose a general action measure for action rule mining as following:

Definition 1 Suppose $P_{f}$ is a total profit for taking an action a, $P(r)$ is the probability of the rule $r$ 's occurrence, and COST denotes the cost of the action. The action measure $u_{a}$ is defined by:

$$
u_{a}=P_{f} \times P(r)-\sum C O S T
$$

The action meausre $u_{a}$ is really the net profit for the user to take a specific action [34].

For example, consider two association rules: one rule is $M i l k \Rightarrow E g g$, and another rule is $M i l k \Rightarrow$ Bread. Milk $\Rightarrow E g g$ means that a consumer likely wants to buy a bottle of milk and also buy a box of eggs. Milk $\Rightarrow$ Bread shows that a consumer would like to buy a bottle of milk and also wants to buy a bag of bread. Both of these association rules are generated with high support and confidence. The probabilities of two association rules occurrences are $60 \%$ and $55 \%$ respectively.

Suppose there is a promotion strategy for eggs and bread. For a consumer, the total profit of performance of this promotion for eggs and bread are $\$ 4.00$ and $\$ 3.00$ 
respectively, and the total costs generated from this promotion for eggs and bread are $\$ 1.00$ and $\$ 1.50$ respectively. According to the action measure $u_{a}$, we know that:

- $u_{a}($ Milk $\Rightarrow E g g)=\$ 4.00 \times 60 \%-\$ 1.00=\$ 1.40$

- $u_{a}($ Milk $\Rightarrow$ Bread $)=\$ 3.00 \times 55 \%-\$ 1.50=\$ 0.15$.

Therefore, we can conclude that $u_{a}(M i l k \Rightarrow E g g)$ is larger than $u_{a}(M i l k \Rightarrow$ Bread) and the rule of $M i l k \Rightarrow E g g$ is more actionable than the rule of $M i l k \Rightarrow$ Bread.

In the real world, there exists many ways to define this action measure concretely and substantially. The construction of action measure is related to the process of decision making and decision theory $[18,21]$.

\subsubsection{Association and Correlation Measures}

Many quantitative measures are proposed to evaluate correlation and association relationships between attribute sets of a discovered rule [46, 67]. In this section, we investigate some quantitative measures for association and correlation. Based on our analysis, we show that each measure reflects various and specific feature(s) of a discovered rule and application domains.

In data mining, discovered knowledge generated from a large information table is often expressed in terms of a set of rules [16]. Yao and Zhong provided an analysis of quantitative measures on discovered rules based on $2 \times 2$ contingency tables [87]. Similar work was done by Tan et al. [67, 68]. This work examined several measures 
Table 2.2: Contingency table for concepts $\phi$ and $\psi$

\begin{tabular}{|c|c|c|c|}
\hline & $\psi$ & $\neg \psi$ & Totals \\
\hline$\phi$ & $|m(\phi) \cap m(\psi)|$ & $|m(\phi) \cap m(\neg \psi)|$ & $|m(\phi)|$ \\
$\neg \phi$ & $|m(\neg \phi) \cap m(\psi)|$ & $|m(\neg \phi) \cap m(\neg \psi)|$ & $|m(\neg \phi)|$ \\
\hline Totals & $|m(\psi)|$ & $\mid m(\neg \psi \mid$ & $|U|$ \\
\hline
\end{tabular}

$(|\cdot|$ denotes the cardinality of a set. $)$

Table 2.3: Contingency table with cardinality

\begin{tabular}{|c|c|c|c|}
\hline & $\psi$ & $\neg \psi$ & Totals \\
\hline$\phi$ & $a$ & $b$ & $a+b$ \\
$\neg \phi$ & $c$ & $d$ & $c+d$ \\
\hline Totals & $a+c$ & $b+d$ & $a+b+c+d=n$ \\
\hline
\end{tabular}

For two concept $(\phi, m(\phi))$ and $(\psi, m(\psi))$, $a=|m(\phi \cap \psi)|, b=|m(\phi \cap \neg \psi)|$, $c=|m(\neg \phi \cap \psi)|, d=|m(\neg \phi \cap \neg \psi)|$.

from statistics, machine learning and data mining fields. Furthermore, both of them found that different measures reflect different application domains or features of discovered rules. Without reference to its usage or users, a measure can not be judged as a better or right one.

We use the sample contingency tables shown in Table 2.2 and 2.3 to analyze several measures on association of the discovered rules.

Given two concepts $(\phi, m(\phi))$ and $(\psi, m(\psi)) . \phi$ and $\psi$ are the intentions (sets of attributes) of the two concepts and used to simply denote the two concept as $\phi$ and $\psi$. 
$m(\phi)$ and $m(\psi)$ are the extensions (sets of objects) of the concepts. A (discovered) rule can be described as a connection or relationship between two concepts and expressed by $\phi \Rightarrow \psi$. The extensions of two concepts can be used to quantitatively measure the degree of the relationship of the rule.

Confidence is a common used measure for evaluating association of a rule. It is a conditional probability $P(\psi \mid \phi)[1,6]$. Based on Table 2.3 , Confidence is defined by:

$$
P(\phi \mid \psi)=\frac{|m(\phi) \cap m(\psi)|}{|m(\psi)|}=\frac{P(\phi, \psi)}{P(\phi)}=\frac{a}{a+b}
$$

where $P(\phi)$ is called support of $\phi$ and is defined by:

$$
P(\phi)=\frac{|m(\phi)|}{|U|}=\frac{a+b}{n}
$$

The support of $\phi$ and $\psi$ occurring together is defined by:

$$
P(\phi, \psi)=\frac{|m(\phi) \cap m(\psi)|}{|U|}=\frac{a}{a+b+c+d}=\frac{a}{n}
$$

The basic idea of confidence is described as the probability that concept $\psi$ occurs given that concept $\phi$ occurs. Two concepts $\phi$ and $\psi$ are viewed as being nonassociative or independent if the occurrence of $\phi$ does not alter the probability of $\psi$ occurring. In other words, if the occurrence of $\phi$ can affect the probability of $\psi$, then we say that the concept $\psi$ is dependent on or associated with the concept $\phi$. Typically, a rule with high support and high confidence are realized that it has a strong association relationship between two concepts and are thought as an interesting or useful rule. 
Confidence is one-direction from $\phi$ to $\psi$ and can be viewed as a one-way association measure [87]. In other words, the concept $\phi$ depends on the concept $\psi$, but $\psi$ may not depend on $\phi$.

Rule-Interest is also a measure on the association of a discovered rule [51]. Based on Table 2.3 , this measure is defined by:

$$
R I=P(\phi, \psi)-P(\phi) P(\psi)=\frac{a^{2}-(a+b)(a+c)}{n^{2}} .
$$

The two concepts $\phi$ and $\psi$ are recognized as being nonassociative or independent when $R I=0(P(\phi) P(\psi)=P(\phi, \psi))$. In fact, this measure determines the degree of association of a rule by comparison of the joint probability of two concepts $P(\phi, \psi)$ with respect to the expected probability of the non-association assumption $P(\phi) P(\psi)$. $R I>0$ represents a positive association from $\phi$ to $\psi . R I<0$ represents a negative association, which is from $\psi$ to $\phi$.

Independence (IND) is similar to the measure of rule-interest $[7,8,63,87]$. Based on Table 2.3, this measure is defined by:

$$
I N D=\frac{P(\phi, \psi)}{P(\phi) P(\psi)}=\frac{a n}{(a+b)(a+c)} .
$$

The two concepts $\phi$ and $\psi$ are recognized as being nonassociative or independent when $I N D=1(P(\phi) P(\psi)=P(\phi, \psi))$. This measure is the ratio of the joint probability of $\phi \cap \psi$ and the probability obtained if $\phi$ and $\psi$ are assumed to be independent. In other words, the rule has a stronger association if the joint probability is further away from the probability under independence [87]. 
IS Measure is another similar measure with respect to the measure of ruleinterest [67]. It can be defined by:

$$
I S=\frac{P(\phi, \psi)}{\sqrt{P(\phi) P(\psi)}}=\sqrt{P(\phi \mid \psi) P(\psi \mid \phi)}=\frac{a}{\sqrt{(a+b)(a+c)}} .
$$

The basic notion of $I S$ measure is similar to the measure of independence IND. Furthermore, it is equivalent to the geometric mean of confidences of the rule. However, its range is between 0 and 1 instead of $I N D$ 's range, between 0 and $\infty$.

Rule-Interest, Independence, and IS measures are symmetric and viewed as twoway association measures [87]. If two concepts $\phi$ and $\psi$ in a rule have a two-way association relationship, then the concept $\phi$ must depends on or are associated with the concept $\psi$, and the converse is also true.

Brin et al. proposed the use of Chi-square $\left(\chi^{2}\right)$ probability testing to evaluate the association between two concepts in a discovered rule [7,63]. If the chi-squared value is 0 , the concepts are really independent, otherwise, they are dependent on each other. However, $\chi^{2}$ testing does not give the strength of association between concepts in a discovered rule [67]. Instead, it only can decide whether concepts in a rule are nonassociative or associative. Therefore, it can not be used to rank the discovered rules. Liu et al. suggested to prune insignificant rules by using the standard $\chi^{2}$ test combined with a support-confidence test [36]. Based on our analysis, the $\chi^{2}$ test can be used to examine the hypothesis of association between the concepts, and the one-way or two-way measures can evaluate the strength of association of concepts in 
Table 2.4: An information table of sales force versus yearly sales revenue

\begin{tabular}{|c|c|c|}
\hline Year & Salesmen & Revenue (Million dollars) \\
\hline 1990 & 15 & 1.35 \\
1991 & 19 & 1.64 \\
1992 & 23 & 2.32 \\
1993 & 22 & 2.31 \\
1994 & 25 & 2.64 \\
1995 & 29 & 2.96 \\
1996 & 31 & 3.21 \\
1997 & 32 & 3.26 \\
1998 & 36 & 3.85 \\
1999 & 39 & 4.03 \\
\hline
\end{tabular}

a rule. Specifically, the Rule-Interest measure is useful to present the direction of the association between concepts.

Besides association, correlation is another common relationship between concepts in a discovered rule. A statistical measure, called correlation coefficient, can compute the degree of this type of relationship between concepts $[46,67]$.

For a discovered rule $\phi \Rightarrow \psi$, we suppose that there exists two value sets $V_{\phi}$ and $V_{\psi}$ in an information table for the two concepts. If $V_{\psi}$ increases or decreases as $V_{\phi}$ increases, then the two concepts are recognized as being closely correlated. For example, Table 2.4 and Figure 2.4.2 demonstrate a strong linear correlation relationship between size of the sales force and yearly sales revenue for a sales department in some companies. The value of revenue, $y$, increases as the number of salesmen, $x$, increases. 


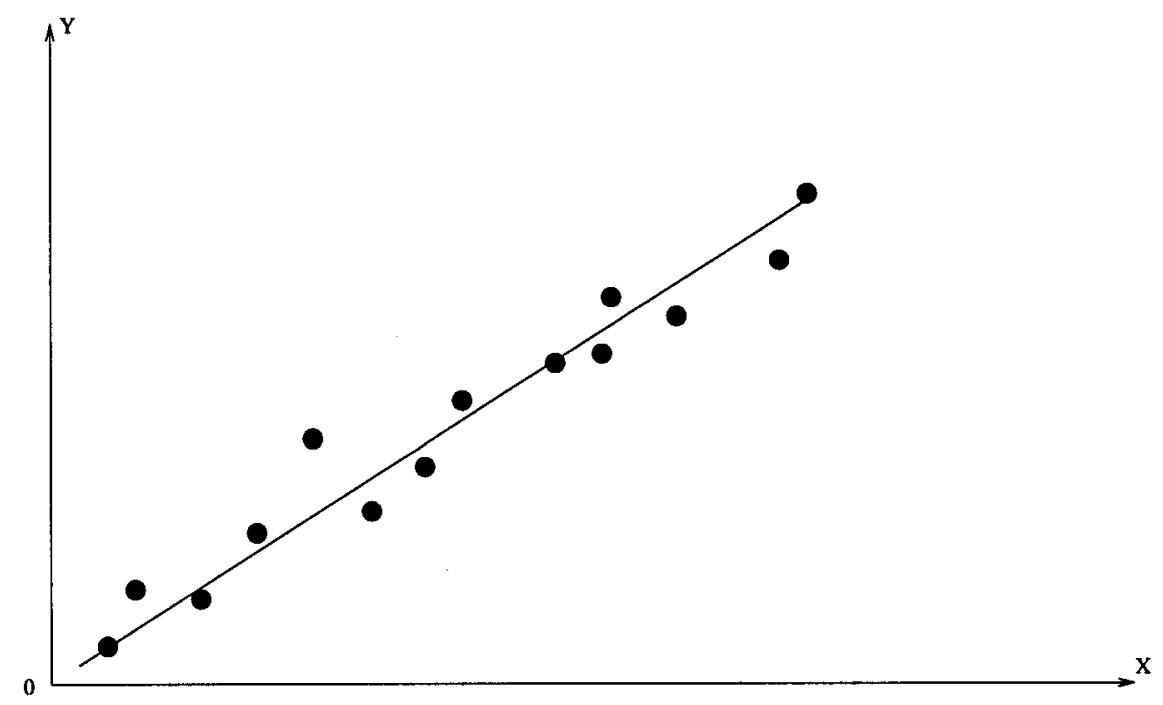

Figure 2.1: A diagram of correlation between two concepts $X$ and $Y$.

The correlation coefficient $r$ on two concepts $\phi$ and $\psi$ is defined by:

$$
r(\phi, \psi)=\frac{S S_{\phi \psi}}{\sqrt{S S_{\phi} S S_{\psi}}}
$$

where $S S_{\phi}=\sum(x-\bar{x})^{2}, x \in V_{\phi}, \bar{x}$ is mean value of $x[46] . S S_{\psi}=\sum(y-\bar{y})^{2}, y \in V_{\psi}$, $\bar{y}$ is mean value of $y . S S_{\phi \psi}=\sum(x-\bar{x})(y-\bar{y})$ is the covariance between concepts $\phi$ and $\psi \cdot \sqrt{S S_{\phi}}$ and $\sqrt{S S_{\psi}}$ are the standard deviations of the real values with respect to the mean value. By extending this equation, we can have:

$$
\begin{aligned}
r(\phi, \psi) & =\frac{S S_{\phi \psi}}{S S_{\phi} S S_{\psi}}=\frac{n \sum x y-\sum x \sum y}{\sqrt{\left[n \sum x^{2}-\left(\sum x\right)^{2}\right]\left[n \sum y^{2}-\left(\sum y\right)^{2}\right]}} \\
& =\frac{E(\phi \wedge \psi)-E(\phi) E(\psi)}{\sigma_{\phi} \sigma_{\psi}}
\end{aligned}
$$

where $E(\phi \wedge \psi)=n \sum x y, E(\phi)=\sum x$, and $E(\psi)=\sum y$ are expected values on concept $\phi \wedge \psi, \phi$, and $\psi$ respectively. $\sigma_{\phi}$ and $\sigma_{\psi}$ denote the standard deviations. 
The correlation coefficient is a number between 0 and 1 . The closer the correlation coefficient to 1 , the stronger the correlation between concepts. If the two concepts are not correlated, $r(\phi, \psi)$ is zero because of $S S_{\phi \psi}=0$ and $E(\phi, \psi)=E(\phi) E(\psi)$. In other words, if $\phi$ and $\psi$ are not closely related to each other, they do not "co-vary", meaning the covariance is small and the correlation is small. If $\phi$ and $\psi$ are very closely related, the covariance is almost the same as $\sigma_{\phi} \cdot \sigma_{\psi}$ and the correlation is almost 1 .

However, correlated concepts may not necessarily be dependent or associated. For example, as illustrated in Table 2.5, $X$ and $Y$ are correlated because $X$ increases as $Y$ increases. However, they are not very associated because the probability of occurring together is $40 \%$, not very high. Also, concepts that are associated may not necessarily be correlated. An example is demonstrated in Table 2.6. The probability of $X$ and $Y$ occurring together is $80 \%$, very high, but, they are not correlated because there is not covariance regularity between $X$ and $Y$. Mari and Kotz analyzed several common and different features of association and correlation measures from the statistical point of view [45]. Correlation coefficient only evaluates the linear relationship between concepts, but there are situations in which linear correlation do not exist but a strong nonlinear association exists between concepts. 
Table 2.5: An example of information table

\begin{tabular}{|c|c|c|}
\hline No. & $\mathrm{X}$ & $\mathrm{Y}$ \\
\hline 1 & 1 & 0 \\
2 & 1 & 0 \\
3 & 1 & 0 \\
4 & 1 & 0 \\
5 & 1 & 0 \\
6 & 1 & 0 \\
7 & 2 & 1 \\
8 & 2 & 1 \\
9 & 3 & 2 \\
10 & 3 & 2 \\
\hline
\end{tabular}

Table 2.6: Another example of information table

\begin{tabular}{|c|c|c|}
\hline No. & $\mathrm{X}$ & $\mathrm{Y}$ \\
\hline 1 & 1 & 0 \\
2 & 1 & 1 \\
3 & 2 & 0 \\
4 & 1 & 3 \\
5 & 3 & 0 \\
6 & 3 & 2 \\
7 & 1 & 1 \\
8 & 2 & 1 \\
9 & 3 & 2 \\
10 & 2 & 2 \\
\hline
\end{tabular}




\subsubsection{Deviation and Change Measures}

In KDD, some measures for rule changes or deviations are proposed and can be classified into two main categories $[4,5,11,23,29,37,39,71,76,77]$. One is to find specific trends, and the other is to discover undesirable changes. Each measure focuses on a specific application domain. Furthermore, an evaluation of changes is relative to previous known knowledge or user beliefs [29]. Some discussions and analysis of evaluation of changes are provided by some researchers $[3,52]$. In this section, we summarize existing studies of measuring rule changes and propose two general measures on changes of discovered rules. The results show that each measure can be applied based on users or application domains.

For a discovered rule $\phi \Rightarrow \psi$, let $\alpha$ denote various types of uncertainties or properties for the rule, such as association (support, confidence) or correlation. Changes of a rule are represented by changes of uncertainty or property $\alpha$. A measure, called change-rate, can be formally defined as following:

Definition 2 For a rule $\phi \Rightarrow \psi$, if its uncertainty or property $\alpha$ is changed to $\alpha^{\prime}, a$ change rate measure can be defined by:

$$
M\left(\alpha, \alpha^{\prime}\right)=\frac{\left|\alpha-\alpha^{\prime}\right|}{\alpha}
$$

This change rate measure is a type of ratio in which the difference between $\alpha$ and $\alpha^{\prime}$ divides the original $\alpha$. The basic idea of this measure is used into the unexpectedness measure [61, 62]. 
Table 2.7: A table of confidence distributions of association rules

\begin{tabular}{|c|c|c|}
\hline$R$ & $\alpha$ & $\alpha^{\prime}$ \\
\hline$R_{1}$ & 0.70 & 0.80 \\
$R_{2}$ & 0.86 & 0.83 \\
$R_{3}$ & 0.65 & 0.77 \\
$R_{4}$ & 0.76 & 0.89 \\
$R_{5}$ & 0.90 & 0.97 \\
\hline
\end{tabular}

For example, the Table 2.7 is about several confidence (conditional probability) distributions on five association rules. The association rules are denoted as $R_{1}, R_{2}$, $\ldots, R_{5} . \alpha$ and $\alpha^{\prime}$ represent the two sets of confidence distributions for five rules. The change-rate measure between $\alpha$ and $\alpha^{\prime}$ on rule $R_{1}$ is $M\left(\alpha, \alpha^{\prime}\right)=\frac{|0.70-0.80|}{0.70}=\frac{1}{7}$.

If there exists a maximum value $\alpha_{\max }$ and a minimum value $\alpha_{\min }$, the change-rate measure can be defined in another way:

Definition 3 For a discovered rule, the change-rate measure between $\alpha$ and $\alpha^{\prime}$ is:

$$
\begin{aligned}
& M\left(\alpha, \alpha^{\prime}\right)=\frac{\alpha^{\prime}-\alpha}{\alpha_{\max }-\alpha}, \quad \text { if } \quad \alpha^{\prime} \geq \alpha \text { and } \alpha \neq \alpha_{\max } \\
& M\left(\alpha, \alpha^{\prime}\right)=\frac{\alpha-\alpha^{\prime}}{\alpha-\alpha_{\min }}, \quad \text { if } \quad \alpha^{\prime} \leq \alpha \text { and } \alpha \neq \alpha_{\min }
\end{aligned}
$$

In this definition, $\alpha-\alpha^{\prime}$ and $\alpha^{\prime}-\alpha$ represent the absolute difference between $\alpha$ and $\alpha^{\prime}$. The change rate measure is a ratio between the absolute difference and the increasing size or decreasing size on $\alpha$. This measure is used in expert system [9].

For example, in the same Table $2.7, \alpha$ on the rule $R_{1}$ is the probability of 0.70 . Since the maximum value of probability is 1.00 and the minimum value of probability 
is 0.00 , the increase size of the rule $R_{1}$ is $1.00-0.70=0.30$, and its decrease size is $0.70-0.00=0.70$. The change rate from $\alpha$ to $\alpha^{\prime}$ on $R_{1}$ is $\frac{0.80-0.70}{1.00-0.70}=\frac{1}{3}$. In the same way, the change rate on $R_{2}$ is $\frac{0.86-0.83}{0.86-0.00}=\frac{3}{86}$.

\subsubsection{Single Rule and Multiple Rules}

The measures of rule interestingness evaluation can also be classified into measures for a single rule and measures for a set of rules. Furthermore, a measure for a set of rules can be obtained from measures for single rules. For example, conditional probability can be used as a measure for a single classification rule, and conditional entropy, which is defined by conditional probability, can be used as a measure for a set of classification rules [80].

Measures for multiple rules concentrate on properties of a set of rules. They are normally expressed as some kinds of average. Hilderman and Hamilton examined many measures for multiple rules known as summaries [26, 27].

\subsubsection{Axiomatic Approaches}

Studies related to rule interestingness evaluation can be generally classified into two classes. One class, the majority of studies, deals with the applications of quantitative measures to reduce the size of search space of rules in the mining process, to filter out mined but non-useful rules, or to evaluate the effectiveness of a data 
mining system. The other class, only a small portion of studies, is devoted solely to the investigations of rule interestingness evaluation on its own. Axiomatic approaches study the required properties of quantitative measures.

Suppose that the discovered knowledge is represented in terms of rules of the form $\phi \Rightarrow \psi$. A quantitative measure of rule $\phi \Rightarrow \psi$ may be computed as a function of support $(\phi)$, support $(\psi)$, support $(\phi \wedge \psi)$, and rule complexity. For the evaluation of rule interestingness, Piatetsky-Shapiro [51] introduced three axioms. Major and Mangano [43] added a fourth axiom. Klösgen studied a special class of measures that are characterized by two quantities, confidence $(\phi \Rightarrow \psi)$ and $\operatorname{support}(\phi)[30]$. The $\operatorname{support}(\psi \wedge \phi)$ is obtained by confidence $(\phi \Rightarrow \psi) \operatorname{support}(\phi)$. Suppose $\operatorname{support}(\phi, \psi)$ is a measure associated with rule $\phi \Rightarrow \psi$. The four axioms are:

(i). $Q(\phi, \psi)=0$ if $\phi$ and $\psi$ are statistically independent,

(ii). $Q(\phi, \psi)$ monotonically increases in confidence $(\phi \Rightarrow \psi)$ for a fixed support $(\phi)$,

(iii). $Q(\phi, \psi)$ monotonically decreases in $\operatorname{support}(\phi)$ for a fixed $\operatorname{support}(\phi \wedge \psi)$,

(iv). $Q(\phi, \psi)$ monotonically increases in support $(\phi)$ for a fixed confidence $(\phi \Rightarrow \psi)>$ $\operatorname{support}(\psi)$

An axiomatic study of measures for multiple rules has been given by Hilderman and Hamilton [26].

The axioms are studied to analyze the properties of some specific quantitative 
measures. Furthermore, this approach focuses on mathematical point of view. Therefore, it can not be a general and unified framework to interpret and compare most measures.

\subsection{Summary}

From the previous discussions, one can make several useful observations. Studies on rule interestingness evaluation can be classified in several ways. Each of them provides a different view and plays different roles in KDD. Most studies on this rule interestingness evaluation concentrate on specific measures and each measure reflects certain features of rules. Quantitative measures are typically interpreted using intuitively defined notions, such as novelty, usefulness, and non-trivialness, unexpectedness, and so on. Therefore, there is a need for a unified framework that enables us to define, interpret, and compare different measures.

A very interesting research direction for rule interestingness evaluation is the study of its foundations. The meaningfulness of a measure should be linked to its usage and roles in KDD. It is also necessary to build a framework in which various notions of rule interestingness evaluation can be formally and precisely defined and interpreted. The user preference should be considered into this framework so that meaningfulness of a measure can be formally defined, interpreted, and compared. This work also should be connected to the study of foundation of KDD or data mining. 


\section{Chapter 3}

\section{Overview of Measurement Theory}

For completeness, we give a brief review of the basic notions of measurement theory that are pertinent to our discussion. The contents of this section draw heavily from Krantz et al. [31], Roberts [57] and French [21].

\subsection{Basic Ideas of the Measurement Theory}

When measuring an attribute of a class of objects or events, we may associate numbers with the individual objects so that the properties of the attribute are faithfully represented as numerical properties [31]. Assignment of numbers also makes it possible to apply the concepts and theories of mathematics into the measurements [57]. The properties are usually described by certain qualitative relations and operations. Consider an example discussed by Krantz et al. [31].

Suppose we are measuring the lengths of a set $U$ of straight, rigid rods. One 
important property of length can be described by a qualitative relation "longer than". Such a relation can be obtained by first placing two rods, say $a$ and $b$, side by side and adjusting them so that they coincide at one end, and then observing whether $a$ extends beyond $b$ at the other end. We say that $a$ is longer than $b$, denoted by $a \succ b$, if $a$ extends beyond $b$. In this case, we would like to assign numbers $f(a)$ and $f(b)$ with $f(a)>f(b)$ to reflect the results of the comparison. That is, we require that numbers assigned to the individual rods satisfy the condition: for all $a, b \in U$,

$$
a \succ b \Longleftrightarrow f(a)>f(b)
$$

In other words, the qualitative relation "longer than", $\succ$, in the empirical system is faithfully reflected by the quantitative relation "greater than", $>$, in the numerical system. Another property of length is that we can concatenate two or more rods by putting them end to end in a straight line, and compare the length of this set with that of another set. The concatenation of $a$ and $b$ can be written as $a \circ b$. In order to reflect such a property, we require the numbers assigned to the individual rods be additive with respect to concatenation. That is, in addition to condition (3.14), the numbers assigned must also satisfy the following condition: for all $a, b \in U$,

$$
f(a \circ b)=f(a)+f(b)
$$

Thus, concatenation $\circ$ in the empirical system is preserved by addition + in the numerical system. Many other properties of length comparison and of concatenation 
of rods can be similarly formulated. For instance, $\succ$ should be transitive and $\circ$ should be commutative and associative. The numbers assigned must reflect these properties as well. This simple example clearly illustrates the basic ideas of measurement theory, which is primarily concerned with choosing consistent quantitative representations of qualitative systems.

Based on the description of basic notions of measurement theory in the above example, some basic concepts and notations are introduced and the formal definitions and formulations of the theory are reviewed.

\subsection{Relational Systems}

Suppose $U$ is a set. The Cartesian product of $U$ with $U$, denoted $U \times U$, is a set of all ordered pairs $(a, b)$ for $a, b \in U$. A binary relation $R$ on a set $U$, simply denote $(U, R)$, is a subset of the Cartesian product $U \times U$. For example, consider the binary relation "less than" $(<)$ relation on real numbers. An ordered pair $(a, b)$ is in the binary relation if and only if $a<b$. Similarly, "greater than" and "equals" also can be defined as the binary relations on real numbers.

With the set $U$, a function $f: U \rightarrow U$ can also be thought of as a binary relation $(U, R)$. A function $f: U^{n} \rightarrow U$ can be an (n+1)-ary relation $(U, R)$. The functions from $U$ into $U$ are called binary operations, or just operations for short. For example, for addition ( + ), given a pair of real numbers $a$ and $b$, there exists a third real number 
$c$ so that $a+b=c$.

A relational system (structure) is a set together with one or more relations (operations) on a set. That is, a relational system is an ordered $(p+q+1)$-tuple $\mathcal{A}=\left(U, R_{1}, \ldots, R_{p}, \circ_{1}, \ldots, \circ_{q}\right)$, where $U$ is a set, $R_{1}, \ldots, R_{p}$ are (not necessarily

binary) relations on $U$, and $\circ_{1}, \ldots, \circ_{q}$ are binary operations on $U$. If the binary operations are considered as a special type of relation, a relational system can be simply denoted as a $(p+1)$-tuple $\mathcal{A}=\left(U, R_{1}, \ldots, R_{p}\right)$.

If $U$ is the set (or a subset) of real numbers, then such a relational system is called a numerical relational system. As illustrated by the example of rigid rods, for measuring the property of length, we can start with an observed or empirical system $\mathcal{A}$ and seek a mapping into a numerical relational system $\mathcal{B}$, which preserves or faithfully reflects all the properties of the relations and operations in $\mathcal{A}$.

\subsection{Axioms of the Empirical System}

Based on the definitions of the relations and operations in a relation system, we should describe the valid use or properties of these relations and operations in order to find the appropriate corresponding numerical system. Many properties are common to well-defined relations. The consistency properties to be preserved are known as axioms. For example, if $U$ is a set of real numbers and $R$ is the relation of "equality" on $U, R$ is reflexive, symmetric, and transitive. However, if $U$ is the set of people in 
the real world and $R$ is the relation "father of" on $U, R$ is irreflexive, asymmetric, and nontransitive.

The set of axioms characterizing the relations in an empirical system should be complete so that every consistency property for the relations that is required is either in the list or deducible from those in the list [21, 31, 57].

\subsection{Homomorphism of Relational Systems}

Consider two relational systems, an empirical (a qualitative) system $\mathcal{A}=\left(U, R_{1}\right.$, $\left.\ldots, R_{p}, \circ_{1}, \ldots, \circ_{q}\right)$ and a numerical system $\mathcal{B}=\left(\Re, R_{1}^{\prime}, \ldots, R_{p}^{\prime}, \circ_{1}^{\prime}, \ldots, \circ_{q}^{\prime}\right)$. A function $f: U \rightarrow \Re$ is called a homomorphism from $\mathcal{A}$ to $\mathcal{B}$, if, for all $a_{1}, \ldots, a_{r_{i}} \in \mathcal{A}$

$$
R_{i}\left(a_{1}, \ldots, a_{r_{i}}\right) \Longleftrightarrow R_{i}^{\prime}\left(f\left(a_{1}\right), \ldots, f\left(a_{r_{i}}\right)\right), \quad i=1, \ldots, p
$$

and for all $a, b \in \mathcal{A}$,

$$
f\left(a \circ_{j} b\right)=f(a) \circ_{j}^{\prime} f(b), \quad j=1, \ldots, q
$$

The empirical system for the earlier example is denoted by $(U, \succ, 0)$, where $U$ is the set of rigid rods and their finite concatenations, $\succ$ is the binary relation "longer than" and $\circ$ is the concatenation operation. The numerical relation system is $(\Re,>,+)$, where $\Re$ is the set of real numbers, > is the usual "greater than" relation, and + is the arithmetic operation of addition. The numerical assignment $f(\cdot)$ is a homomorphism which maps $U$ into $\Re, \succ$ into $>$, and $\circ$ into + in such a way that $>$ preserves the 
properties of $\succ$, and + preserves the properties of $\circ$ as stated by conditions (3.14) and $(3.15)$.

In general, a measurement has been performed if a homomorphism can be assigned from an empirical (observed) relational system $\mathcal{A}$ to a numerical relational system $\mathcal{B}$. The homomorphism is said to give a representation, and the triple $(\mathcal{A}, \mathcal{B}, f)$ of the empirical relational system $\mathcal{A}$, the numerical relational system $\mathcal{B}$, and the function $f$ is called a scale or measure. Sometimes, a homomorphism from an empirical relational system into the set of real numbers is referred alone as a scale (measure).

Suppose given numerical scales (measures), new scales or measures defined in terms of the old ones are called derived scales or derived measures. For example, density $d$ can be defined in terms of mass $m$ and volume $v$ as $d=m / v$. The density $d$ is the derived scale (measure), and the mass $m$ and volume $v$ are called as primitive scales (measures).

\subsection{Procedure of Measurement}

Generally, there are three fundamental steps in measurement theory $[21,31,57]$. Suppose we are seeking a quantitative representation of an empirical system. The first step, naturally, is to define the relations and operations to be represented, where the axioms of the empirical system are determined. The next task is to choose a numerical system. The final step is to construct an appropriate homomorphism. 
A representation theorem asserts that if a given empirical system satisfies certain axioms, then a homomorphism into the chosen numerical system can be constructed. Another question concerns the uniqueness of the scale. A uniqueness theorem is generally obtained by identifying a set of admissible transformations. If $f(\cdot)$ is a scale representing an empirical system and if $\lambda(\cdot)$ is an admissible transformation, then $\lambda(f(\cdot))$ is also a scale representing the same empirical system.

Applications of measurement theory include the axiomatization of probability and expected utility theory $[18,47,60]$, the axiomatization of possibility functions [13] and the axiomatization of belief functions [74]. 


\section{Chapter 4}

\section{Application of Measurement Theory to Rule Interestingness Evaluation}

Given a database, in theory, there exists a set of rules embedded in it, independent of whether one has an algorithm to mine them. For a particular application, the user may only be interested in a certain type of rules. Therefore, the key issue of rule interestingness evaluation is the measurement of the rule's usefulness or interestingness expressed by a user preference relation. According to measurement theory, we follow its methodologies to establish a framework of measurement of rule interestingness based on user preference relation. 


\subsection{User Preference Relations}

Based on measurement theory, for first step, we should model user judgment or user preference as a kind of binary relation, called a user preference relation. If the user prefers a rule to another rule, then we can say that one rule is more useful or interesting than the other rule.

Assume we are given a set $\mathbf{R}$ of discovered rules. Since the usefulness or interestingness of rules should be judged by users, we focus on user preference as a binary relation on the set of discovered rules. Given two rules $r^{\prime}, r^{\prime \prime} \in \mathbf{R}$, if a user judges $r^{\prime}$ to be more useful than $r^{\prime \prime}$, we say that the user prefers $r^{\prime}$ to $r^{\prime \prime}$ and denote it by $r^{\prime} \succ r^{\prime \prime}$. That is,

$$
r^{\prime} \succ r^{\prime \prime} \Leftrightarrow \text { the user prefers } r^{\prime} \text { to } r^{\prime \prime} \text {. }
$$

In the absence of strict preference, i.e., if both $\neg\left(r^{\prime} \succ r^{\prime \prime}\right)$ and $\neg\left(r^{\prime} \succ r^{\prime \prime}\right)$ hold, we say that $r^{\prime}$ and $r^{\prime \prime}$ are indifferent. An indifference relation $\sim$ on $\mathbf{R}$ can be defined as follows:

$$
r^{\prime} \sim r^{\prime \prime} \Leftrightarrow\left(\neg\left(r^{\prime} \succ r^{\prime \prime}\right), \neg\left(r^{\prime \prime} \succ r^{\prime}\right)\right) .
$$

With the user preference relation, the empirical relational system can be defined as following:

Definition 4 Given a set of discovered rules $\mathbf{R}$ and user preference relation $\succ$, the pair $(\mathbf{R}, \succ)$ is called the (empirical) relational system of the set of discovered rules. 
The user judgement on rules is formally described by a user preference relation $\succ$ on $\mathbf{R}$. The next issue is to identify the desired properties of a preference relation so that it can be measured quantitatively.

\subsection{Axioms of User Preference Relation}

The consistency properties are known as axioms. Based on measurement theory, we consider the following two axioms:

\section{- Asymmetry:}

$$
r^{\prime} \succ r^{\prime \prime} \Rightarrow \neg\left(r^{\prime \prime} \succ r^{\prime}\right),
$$

- Negative transitivity:

$$
\left(\neg\left(r^{\prime} \succ r^{\prime \prime}\right), \neg\left(r^{\prime \prime} \succ r^{\prime \prime \prime}\right)\right) \Rightarrow \neg\left(r^{\prime} \succ r^{\prime \prime \prime}\right) .
$$

The first axiom requires that a user cannot prefer $r^{\prime}$ to $r^{\prime \prime}$ and at the same time prefers $r^{\prime \prime}$ to $r^{\prime}$. In other words, the result of a user preference on two different discovered rules is not contradiction. In fact, this axiom ensures the user preference or user judgement is rational. The second is the negative transitivity axiom, which means that if a user does not prefer $r^{\prime}$ to $r^{\prime \prime}$, nor $r^{\prime \prime}$ to $r^{\prime \prime \prime}$, the user should not prefer $r^{\prime}$ to $r^{\prime \prime \prime}$. These two axioms present the essential properties of a user preference relation. In other words, on one hand, a binary relation that satisfies these two axioms might be deemed as a user preference relation. On the other hand, a user preference relation 
must satisfy these two axioms.

If a user preference relation is a weak order [57], it is transitive, i.e., $r^{\prime} \succ r^{\prime \prime}$ and $r^{\prime \prime} \succ r^{\prime \prime \prime}$ imply $r^{\prime} \succ r^{\prime \prime \prime}$. It seems reasonable that a user preference relation should satisfy these two axioms.

A few additional properties of a weak order are summarized in the following lemma $[18,57,78]$ :

Lemma 1 Suppose a preference relation $\succ$ on a finite set of rules $\mathbf{R}$ is a weak order. Then,

- the relation $\sim$ is an indifference relation,

- exactly one of $r^{\prime} \succ r^{\prime \prime}, r^{\prime \prime} \succ r^{\prime}$ and $r^{\prime} \sim r^{\prime \prime}$ holds for every $r^{\prime}, r^{\prime \prime} \in \mathbf{R}$.

- the relation $\succ^{\prime}$ on $\mathbf{R} / \sim$ defined by $X \succ^{\prime} Y \Leftrightarrow \exists r^{\prime}, r^{\prime \prime}\left(r^{\prime} \succ r^{\prime \prime}, r^{\prime} \in X, r^{\prime \prime} \in Y\right)$, is a linear order, where $X$ and $Y$ are elements of $\mathbf{R} / \sim$.

where $\mathbf{R} / \sim$ denotes the set of partitions generated by the indifference relation $\sim$ on the set of discovered rules $\mathbf{R}$.

The proofs of this lemma have been presented by Roberts and Fishburn [18, 57]. This lemma ensures the other properties of a user preference relation as a weak order.

A linear order is a weak order in which any two different elements are comparable. This lemma implies that if $\succ$ is a weak order, the indifference relation $\sim$ divides the set of rules into disjoint subsets. In other words, based on a user preference relation, 
the set of discovered rules can be partitioned into several equivalence groups. The rules in a group are viewed as indifference according to the user preference.

After defining a user preference relation $\succ$ on a set of discovered rules $\mathbf{R}$ and identifying the necessary axioms of $\succ$, we should seek a quantitative representation of the user preference relation. In measurement-theoretic terminology, the requirement of a weak order indeed suggests the use of an ordinal numbers as a quantitative representation $[57,78]$. Then we should construct a homomorphism from the set of discovered rules into the ordinal numbers.

\subsection{Homomorphism based on Real-valued Function}

A representation theorem is proposed by Roberts and Pfanzagl $[57,50]$. We follow their works to present our homomorphism. A homomorphism for the measurement of user preference of rules is shown by the following representation theorem [18, 57]:

Theorem 1 Suppose $\mathbf{R}$ is a finite non-empty set of rules and $\succ$ a preference relation on $\mathbf{R}$. There exists a real-valued function $u: \mathbf{R} \longrightarrow \Re$ satisfying the condition,

$$
r^{\prime} \succ r^{\prime \prime} \Leftrightarrow u\left(r^{\prime}\right)>u\left(r^{\prime \prime}\right),
$$

if and only if $\succ$ is a weak order. Moreover, $u$ is defined as a strictly monotonic increasing transformation. 
The proof of this theorem has been provided by Roberts and Fishburn $[18,57]$. The important point is that Theorem 1 provides a direct link between the notion of rule ranking and that of a user preference relation.

The numbers $u\left(r^{\prime}\right), u\left(r^{\prime \prime}\right), \ldots$ as ordered by $>$ reflect the order of $r^{\prime}, r^{\prime \prime}, \ldots$ under $\succ$. The function $u$ is referred to as an order-preserving utility function. It quantifies a user preference relation and provides a measurement of user judgments. According to Theorem 1, the axioms of a weak order are the conditions which allow the measurement. Thus, to see if we can measure a user's preference to the extent of producing an ordinal utility function, we just check if this preference satisfies the conditions of asymmetry and negative transitivity. A rational user's judgments must allow the measurement in terms of a quantitative utility function. On the other hand, another interpretation treats the axioms as testable conditions. Whether we can measure the user judgments depends on whether the user preference relation is a weak order [78].

\subsection{Ordinal Measurement of Rules Interestingness}

In the above discussion, only the asymmetry and negative transitivity axioms must be satisfied. This implies that the ordinal measure is used for the measurement of user preference. For the ordinal measure, it is meaningful to examine the order or compare the order induced by the utility function.

The main ideas can be illustrated by a simple example. Suppose a user preference 
relation $\succ$ on a set of rules $\mathbf{R}=\left\{r_{1}, r_{2}, r_{3}, r_{4}\right\}$ is specified by the following weak order:

$$
r_{3} \succ r_{1}, \quad r_{4} \succ r_{1}, \quad r_{3} \succ r_{2}, \quad r_{4} \succ r_{2}, \quad r_{4} \succ r_{3} .
$$

We assume that this relation $\succ$ satisfies the asymmetry and negative transitivity conditions (axioms). We can find three equivalence classes $\left\{r_{4}\right\},\left\{r_{3}\right\}$, and $\left\{r_{1}, r_{2}\right\}$. Furthermore, they can be arranged as three levels:

$$
\left\{r_{4}\right\} \succ^{\prime}\left\{r_{3}\right\} \succ^{\prime}\left\{r_{1}, r_{2}\right\}
$$

We can defined the utility function $u_{1}$ as follows:

$$
u_{1}\left(r_{1}\right)=0, \quad u_{1}\left(r_{2}\right)=0, \quad u_{1}\left(r_{3}\right)=1, \quad u_{1}\left(r_{4}\right)=2 .
$$

If there exists another utility function $u_{2}$ that may be used as following:

$$
u_{2}\left(r_{1}\right)=5, \quad u_{2}\left(r_{2}\right)=5, \quad u_{2}\left(r_{3}\right)=6, \quad u_{2}\left(r_{4}\right)=7
$$

then we say that the two utility functions $u_{1}$ and $u_{2}$ are equivalent because they preserve the same order for any pair of rules, although they use different values.

After construction of the homomorphism, we establish a framework of measurement for rule interestingness evaluation. Based on this formal framework of measurement, we can study different types of user preference relations. In order to do so, we need to impose more axioms on the user preference relation. The axioms on user preference relations can be easily interpreted and related to domain specific notions. 
Based on the framework, we know that an individual measure in accordance with a user preference reflects a specific perspective or feature of rules. However, in the real world, when evaluating the discovered rules, several features of the rules are considered at the same time. In the next section, we introduce a linear model for the combination of measures and then apply an inductive learning method for finding the coefficients of the linear function.

\subsection{An Inductive Learning Procedure for Linear Combina- tion Measure}

From the review of existing measures for rule interestingness in Chapter 2, one can see that most measures focus on specific user preferences and each reflects a certain feature of the rules, such as dependency, unexpectedness, profit, and others. In the real world, people would like to consider several features of rules at the same time. In other words, a user preference may be determined by a combination of several specific user preferences. The measure to represent this user preference is a combination of several specific measures. For example, when evaluating a rule, a user may not only estimate the unexpectedness of the rule, but also compute and measure the profits generated by applying the rule. The rule is deemed as interesting if it is more unexpected and generates more profits from applications. In this case, the user preference is represented by combining two specific preferences (unexpectedness 
and profit) and the corresponding measure must be a combination of the measures of unexpectedness and profit.

In rest of this section, we formally define a linear model for rule interestingness evaluation and apply an inductive learning procedure to find the coefficients for the linear function based on a training set of discovered rules.

Let $\mathbf{R}$ be a finite set of discovered rules and $\succ$ be a user preference relation on $\mathbf{R}$. For a training set of rules $\mathbf{S} \subseteq \mathbf{R}$, we assume that the user fully specifies her or his preference by examining all the rules. Furthermore, the user preference relation $\succ$ must satisfy two axioms of asymmetry and negative transitivity. That is, $\succ$ is weak order in order to ensure that the user preference is rational. Generally, by using the ordered rules in the training set $\mathbf{S}$, one can possibly infer a linear function (measure) by applying an inductive learning method.

A simple combination of multiple measures is a linear combination. The formal definition of linear measure is given in the following.

Definition 5 Given $n$ measures $\mathbf{m}=\left\{m_{1}, m_{2}, \ldots, m_{n}\right\}$, their linear combination ( $a$ linear measure) is given by product of a weight vector $\mathbf{w}$ and the measure vector $\mathbf{m}(r)$ :

$$
\mathbf{M}(r)=w_{0}+\mathbf{w} \cdot \mathbf{m}(r)=w_{0}+w_{1} m_{1}(r)+w_{2} m_{2}(r)+\ldots+w_{n} m_{n}(r)
$$

where $\mathbf{w}$ is a finite set of weights $\left\{w_{1}, w_{2}, \ldots, w_{n}\right\}$ and $w_{0}$ is a given threshold.

According to the representation theorem (Theorem 1), for two rules $r$ and $r^{\prime}$ in a 
training set, we can have:

$$
r \succ r^{\prime} \Longleftrightarrow \mathbf{M}(r)>\mathbf{M}\left(r^{\prime}\right) \Longleftrightarrow \mathbf{w} \cdot \mathbf{m}(r)>\mathbf{w} \cdot \mathbf{m}\left(r^{\prime}\right)
$$

if and only if $\succ$ is a weak order and $\mathbf{M}$ is a strictly monotonic increasing real-valued function.

Unfortunately, this theorem does not say how one can construct a utility function (measure) with a collection of discovered rules. In order to infer a linearly combined measure $\mathbf{M}$ from a training set of rules, we study the class of linear user preference relations.

Definition 6 For a set of discovered rules $\mathbf{R}$ and a user preference relation $\succ$ on $\mathbf{R}$, we say that the preference relation $\succ$ is linear (order) if there exists weight vectors $\mathbf{w}$ such that for any $r, r^{\prime} \in \mathbf{R}$,

$$
r \succ r^{\prime} \Longleftrightarrow \mathbf{w} \cdot \mathbf{m}(r)>\mathbf{w} \cdot \mathbf{m}\left(r^{\prime}\right)
$$

The above definition implies that

$$
\mathbf{w} \cdot \mathbf{m}(r)=\mathbf{w} \cdot \mathbf{m}\left(r^{\prime}\right) \Longleftrightarrow r \sim r^{\prime}
$$

This condition guarantees that in the training set, less preferred rules will not be ranked in front of the preferred ones based on $\mathbf{M}$. Furthermore, rules in the same equivalence class produced by the indifference relation have the same utility value.

According to Definition 5, inductive learning problem of a linearly combined measure $\mathbf{M}$ is indeed of finding the coefficients, weight vector $\mathbf{w}$, of the measure $\mathbf{M}$ with 
respect to a given set of individual measures $\mathbf{m}$. Now, we show how the weight vector can be inferred from a training set of discovered rules $\mathbf{S} \subseteq \mathbf{R}$.

In inductive learning, a function inferred from a training set may not necessarily be accurate when it is applied to all objects in the universe [75]. In other words, the weight vector of the combination measure $\mathbf{M}$ may not necessarily be completely correct when one examines it in the whole set of discovered rules. However, by enlarging the training set, it is expected to produce a more accurate weight vector if the user preference relation is linear. An additional sample is selected from the enlarged training set of rules and then an improved weight vector is produced [14]. This process can be repeated until the user satisfies with the evaluation result generated by the linearly combined measure.

Suppose a user preference relation $\succ$ on a set of discovered rules $\mathbf{R}$ is linear. An inductive procedure to formulate a weight vector $\mathbf{w}$ from a training set of rules $\mathbf{S} \subseteq \mathbf{R}$ is developed as follows.

For two rules $r, r^{\prime} \in \mathrm{S}$ such that $r \succ r^{\prime}$ holds. By Definition 6, we can have $\mathbf{w} \cdot \mathbf{m}(r)>\mathbf{w} \cdot \mathbf{m}\left(r^{\prime}\right)$. Let $\mathbf{b}=\mathbf{m}(r)-\mathbf{m}\left(r^{\prime}\right)$ be a difference vector between two rules. Given a training set of discovered rules $\mathbf{S}$, the set of all difference vectors $\mathbf{B}$ is defined by:

$$
\mathbf{B}=\left\{\mathbf{b}=\mathbf{m}(r)-\mathbf{m}\left(r^{\prime}\right) \mid r, r^{\prime} \in \mathbf{S} \text { and } r \succ r^{\prime}\right\}
$$

Therefore, we know that the problem of seeking a weight vector $\mathbf{w}$ for the combination 
measure $\mathbf{M}$ is equivalent to solving the following linear inequalities:

$$
\mathbf{w} \cdot \mathbf{b}>0, \text { for every } \mathbf{b} \in \mathbf{B} \text {. }
$$

There exists a solution for weight vector $\mathbf{w}$ which satisfies above inequalities. However, there may be more than one solution for a given training set. It is understood that any solution can be accepted by the user if it can produce the satisfying result of measuring. Thus, in this thesis, we focus on how to find such a solution of weight vector.

We adopt a method for solving the linear inequalities based on the minimization of a suitably chosen scalar function. For a weight vector $\mathbf{w}$ and a difference vector $\mathbf{b}=\mathbf{m}(r)-\mathbf{m}\left(r^{\prime}\right) \in \mathbf{B}$, if $\mathbf{w} \cdot \mathbf{b}>0$, then we say that the vector $\mathbf{w}$ correctly satisfies the preference relation between $r$ and $r^{\prime}$. If $\mathbf{w} \cdot \mathbf{b} \leq 0$, then an error occurs. All of errors with respect to the vector $\mathbf{w}$ can be defined by:

$$
\mathbf{J}(\mathbf{w})=\sum_{\mathbf{b} \in \mathbf{E}(\mathbf{w})}-\mathbf{w} \cdot \mathbf{b}
$$

where $\mathbf{E}(\mathbf{w})$ is defined by:

$$
\mathbf{E}(\mathbf{w})=\left\{\mathbf{b}=\mathbf{m}(r)-\mathbf{m}\left(r^{\prime}\right) \mid r, r^{\prime} \in \mathbf{S}, r \succ r^{\prime} \text { and } \mathbf{w} \cdot \mathbf{b} \leq 0\right\} \subseteq \mathbf{B} .
$$

We assume that $\mathbf{J}(\mathbf{w})=0$ if the set of $\mathbf{E}(\mathbf{w})$ is empty, that is, $\mathbf{E}(\mathbf{w})=\emptyset$. Here, $\mathbf{J}(\mathbf{w})$ is nonnegative because of $\mathbf{w} \cdot \mathbf{b} \leq 0 . \mathbf{J}(\mathbf{w})$ is equal to 0 only if $\mathbf{w}$ is a solution vector (i.e., $\mathbf{E}(\mathbf{w})=\emptyset) . \mathbf{J}(\mathbf{w})$ is called the perceptron criterion function in pattern recoginition [14]. 
An iterate procedure can be developed to search for a weight vector that minimizes the function $\mathbf{J}(\mathbf{w})$ and at the same time satisfies the condition $\mathbf{E}(\mathbf{w})=\emptyset$. In this procedure, we start with an arbitrary vector $\mathbf{w}_{\mathbf{0}}$ and compute the corresponding $\sum \mathbf{b} \in \mathbf{E}(\mathbf{w})$. The next vector $\mathbf{w}_{\mathbf{1}}$ is obtained by reducing some distance from $\mathbf{w}_{\mathbf{0}}$, and so on. Generally, the vector $\mathbf{w}_{\mathbf{k}+1}$ can be represented by:

$$
\mathbf{w}_{\mathbf{k}+\mathbf{1}}=\mathbf{w}_{\mathbf{k}}+\alpha \sum_{\mathbf{b} \in \mathbf{E}\left(\mathbf{w}_{\mathbf{k}}\right)} \mathbf{b}
$$

where $\alpha$ is a positive number that predetermined by the user. In this thesis, for simplicity, we assume $\alpha=1$.

Based on the description of searching a weight vector above, we summarize the procedure as following algorithm:

1. Set an initial weight vector $\mathbf{w}_{0}$ and counter $k=0$,

2. $\mathbf{w}_{\mathbf{k}+\mathbf{1}}$ is the weigh vector in the $(k+1)$ th iteration,

3. Set the set of difference vectors:

$$
\begin{aligned}
\mathbf{E}(\mathbf{w}) & =\left\{\mathbf{b}=\mathbf{m}(r)-\mathbf{m}\left(r^{\prime}\right) \mid r, r^{\prime} \in \mathbf{S}, r \succ r^{\prime}\right. \\
& \text { and } \mathbf{w} \cdot \mathbf{b} \leq 0\} \subseteq \mathbf{B} .
\end{aligned}
$$

If $\mathbf{E}(\mathbf{w})=\emptyset$ (i.e., $\mathbf{w}_{\mathbf{k}}$ is a solution vector), stop the procedure.

4. Compute the next weight vector $\mathbf{w}_{\mathbf{k}+\mathbf{1}}$ :

$$
\mathbf{w}_{\mathbf{k}+1}=\mathbf{w}_{\mathbf{k}}+\alpha \sum_{\mathbf{b} \in \mathbf{E}\left(\mathbf{w}_{\mathbf{k}}\right)} \mathbf{b}
$$


5. Let $k=k+1$ and go back to step 2 ,

Duba and Hart discussed a similar procedure to minimize the perceptron criterion function in order to obtain an approximate vector such that it can correctly classify the objects [14]. Wong and Yao proved that if the user preference relation $\succ$ is linear, a solution of weight vector can be found [75]. Main ideas for proving the convergence of the above procedure is to prove that the weight vector $\mathbf{w}_{\mathbf{k}}$ is closer to a solution by correction in each iteration. In other words, if $\mathbf{w}$ is a solution vector, then $\left\|\mathbf{w}_{\mathbf{k}+\mathbf{1}}-\mathbf{w}\right\|$ is smaller than $\left\|\mathbf{w}_{\mathbf{k}}-\mathbf{w}\right\|(\|\cdot\|$ denotes the magnitude of a vector $)$. The conclusion is that $\mathbf{w}_{\mathbf{k}}$ eventually converges to a solution vector after a finite number of iterations.

By adding more sample rules to the training set, the weight vector $\mathbf{w}$ will approach the solution weight vector applicable to the whole collection of discovered rules $\mathbf{R}$. We can use the weight vector $\mathbf{w}$ to construct the combined measure $\mathbf{M}$.

In this thesis, we only consider the linear model of a combined measure. The main advantages of a linear measure are its simplicity and capability of acceptable performance [14]. If the model of combination measure is not linear, the inductive method is not appropriate [75]. One may have to select other methods of learning for the nonlinear model of combination measure.

It should be emphasized that an assumption in the proposed method is that the user preference relation is linear. If in practice one observes nonlinear cases in the training set, our method of inductive learning can not be used and there are 
two reasonable alternatives: (1) one might choose nonlinearly combined measure for the nonlinear user preference relation, or (2) one may consider the assumption of linearity of user preference relation is a first-order approximation. In the latter case, our method of inductive learning can still be used to obtain an approximate weight vector in a combination measure. A detailed analysis of this procedure is provided by Duba and Hart [14].

In the linear model of combined measure, $\mathbf{m}$ represents a finite set of individual measures predetermined and given by the user. Each measure is a quantitative representation of a specific characteristic of the rules. A related problem is how to select those specific measures. The policies of selection of individual measures not only depend on the user but also depend on the relationships between those specific measures, such as dependence or correlation. If two measures depend on or associate with each other, whether one selects them into a combination measure will affect the construction of the combination measure and furthermore influence the inductive learning of the weight vector $\mathbf{w}$. In this thesis, we only assume that those individual measures are given by the user. The topic of selection of particular measures can be a future research work. 


\subsection{Summary}

In this section, we establish a unified framework for measurement of rule interestingness. Measurement theory is used to build this framework with respect to the user preference. The user preference is expressed by a binary relation on a set of discovered rules. A representation theorem ensures that a utility function (measure) can be used to represent the user preference relation if and only if the user preference relation satisfies the conditions.

We show that a linearly combined measure can be inferred by inductive learning procedure based on a training set of discovered rules. A combined measure summarizes the information by several measures and is formally defined by sets of the weights and measures. An inductive learning procedure for finding the weights of the linear measure is introduced and applied. It is demonstrated that the proposed framework is very useful and flexible. One can easily apply results from measurement theory and related fields. 


\section{Chapter 5}

\section{Conclusion and Future Research}

The main contributions of this thesis are providing a review of current studies on rule interestingness evaluation from different perspectives, and proposing a unified and general framework of measurement for rule interestingness evaluation. This framework is established based on user preference by using methodologies of measurement theory. By applying the framework, a combination measure can be inferred by an inductive learning procedure based on a training set of discovered rules ordered by a user preference relation.

\subsection{Summary}

We present an introduction of Knowledge Discovery in Databases and discuss the fundamental issues of evaluation in this thesis. A review of rule evaluation suggests that we can study the topic from different points of views. Each view leads to specific 
perspectives and issues. Some particular quantitative measures, such as association and correlation, are analyzed and discussed explicitly and critically. Furthermore, for changes of discovered rules, two general measures for different application purposes are summarized and explained.

From the review of current studies of rule interestingness evaluation, it is recognized that there is a need of a unified and fundamental framework, where various measures can be defined and interpreted formally and precisely. Furthermore, user preference is also necessary to be considered into this framework.

Measurement theory is used to establish this solid foundation of rule interestingness evaluation. Fundamental issues are discussed based on the user preference. Conditions on a user preference relation are analyzed so that one can obtain a quantitative measure that reflects the user-defined order of rules. User preference, expressed by a binary relation on a set of rules, is used as a primitive notion to model the measurement of rule interestingness. A measure of rule interestingness evaluation is viewed as a quantitative representation of a user preference.

Based on the proposed framework, we introduce a linear combination measure and develop an inductive learning method to infer this linearly combined measure. The training set of discovered rules is ordered by the user preference relation. Furthermore, we discuss the limitations and necessary assumptions for construction of the measure and inductive learning method. It is clarified that our framework is very useful for 
further studies on foundations of rule interestingness evaluation and KDD.

\section{$5.2 \quad$ Future Research}

Rule interestingness evaluation is a broadly researched topic in the KDD field. The proposed framework is a user-centered approach based on the user preference relation and provides a solid basis for future research. We can investigate and identify some additional qualitative properties on the user preference relation.

The study of rule interestingness evaluation needs to be connected to the study of foundations of KDD and data mining. Yao proposed a three-layed framework for a conceptual formulation of KDD [79]. At philosophy level, formal characterization, description, representation, and classification of knowledge embedded in a database can be developed, investigated and formulated. In this thesis, we only emphasized one type of knowledge: $\phi \Rightarrow \psi$. There are lots of other kinds of knowledge that can be pursued, such as high ordered rules [58, 84], and lots of related research that can be done, such as the evaluation of different forms of knowledge and examination of different relationships between concepts by using other methods such as formal concept analysis $[72,73]$.

For the measures of the rule changes, there are still many topics in which we can study further. For example, Plewis analyzed several types of data changes according to the time (age, period, etc.), dimension, location, data sets, or user beliefs [53]. 
Each type of data changes may cause variations of discovered rules, which take place either in the intentions of concepts or the extensions of concepts. Therefore, there is a need to classify different types of changes and explore various useful measures for different application purposes. 


\section{Bibliography}

[1] Agrawal, R., Imielinski, T., and Swami, A. Mining association rules between sets of items in large databases, Proceedings of ACM SIGMOD, 207-216, 1993.

[2] Barber, B. and Hamilton, H. Extracting share frequent itemsets with infrequent subsets, Data Mining and Knowledge Discovery, 7, 153-185, 2003.

[3] Bartholomew, D. The Statistical Approach to Socail Measurement, Academic Press, New York, 1996.

[4] Bay, S. and Pazzani, M. Detecting group differences: mining contrast sets. Proceedings of $K D D, \mathbf{5}, 213-246,2001$.

[5] Bay, S. and Pazzani, M. Detecting change in categorical data: mining contrast sets. Proceedings of KDD, 302-306, 1999.

[6] Bayardo, R.J. and Agrawal, R. Mining the most interesting rules. Proceedings of $K D D, 145-154,1999$. 
[7] Brin, S., Motwani, R., and Silverstein, C. Beyond market baskets: generalizing association rules to correlations, Proceedings of ACM SIGMOD, 265-276, 1997.

[8] Brijs, T., Swinnen, G., Vanhoof, K., and Wets, G. Using association rules for product assortment decisions: a case tudy. Proceedings of KDD, 254-260, 1999.

[9] Buchanan, B. and Shortliffe, E. Rule-Based Expert System: The MYCIN Experiments of the Stanford Heuristic Programming Project, Addison-Wesley, London, 1984.

[10] Chen, M.S., Han, J., and Yu, P.S. Data mining: an overview from database perspective, IEEE Transactions on Knowledge and Data Engineering, 8, 866833, 1996.

[11] Dong, G. and Li, J. Efficient mining of emerging patterns: discovering trends and differences. Proceedings of KDD, 43-52, 1999.

[12] Doyle, J. Rationality and its role in reasoning, Computational Intelligence, 8, 376-409, 1992.

[13] Dubois, D. Belief structures, possibility theory and decomposable confidence measures on finite sets, Computers and Artificial Intelligence, 5, 403-416, 1986.

[14] Duda, R.O. and Hart, P.E. Pattern Classification and Scene Analysis, John Wiley, New York, 1973. 
[15] Facione, P.A., Scherer, D., and Attig, T. Values and Society: An Introduction to Ethics and Social Philosophy, Prentice-Hall, Inc., New Jersey, 1978.

[16] Fayyad, U.M., Piatetsky-Shapiro, G., Smyth, P., and Uthurusamy, R. (Eds.), $A d-$ vances in Knowledge Discovery and Data Mining, AAAI/MIT Press, California, 1996.

[17] Fayyad, U.M., Piatetsky-Shapiro, G., and Smyth, P. From data mining to knowledge discovery in databases, AI Magazine, 17, 37-54, 1996.

[18] Fishburn, P.C. Utility Theory for Decision Making, John Wiley \& Sons, Inc., New York, 1970.

[19] Frawely, W., Piatetsky-Shapiro, G., and Matheus, C. Knowledge discovery in database: an overview, Knowledge Discovery in Database, AAAI/MIT Press, $1-27,1991$.

[20] Freitas, A.A. On rule interestingness measures, Knowledge-Based Systems, 12, 309-315, 1999

[21] French, S. Decision Theory: An Introduction to the Mathematics of Rationality, Ellis Horwood Limited, Chichester, West Sussex, England, 1988.

[22] Gago, P., and Bento, C. A metric for selection of the most promising rules, Proceedings of PKDD, 19-27, (1998) 
[23] Ganti, V., Gehrke, J., and Ramakrishnan, R. A framework for measuring changes in data characteristics. Proceedings of 18th Symposium on Principles of Database Systems, 126-137, 1999.

[24] Geisler, E. Creating Value with Science and Technology, Quorum Books, London, 2001.

[25] Han, J. and Kamber, M. Data mining: Concept and Techniques, Morgan Kaufmann, Palo Alto, CA, 2000.

[26] Hilderman, R.J. and Hamilton, H.J. Knowledge Discovery and Measures of Interest, Kluwer Academic Publishers, Boston, 2001.

[27] Hilderman, R.J. Mining Summarizes from Databases using Domain Generalization Graphs and Objective Measures of Interestingness, Ph.D. Thesis, University of Regina, 2000.

[28] Horvitz, E. and Heckerman, D. The inconsistent use of measures of certainty in artificial intelligence research, in: L.N. Kanal and J.F. Lemmer (Eds.), Uncertainty in Artificial Intelligence, 137-151, 1986.

[29] Hussain, F., Liu, H., Suzuki, E., and Lu, H. Exception rule mining with a relative interestingness measure. Proceedings of PAKDD, 86-97, 2000. 
[30] Klösgen, W. Explora: a multipattern and multistrategy discovery assistant, in: U.M. Fayyad, G. Piatetsky-Shapiro, P. Smyth, and R. Uthurusamy, (Eds.), Advances in Knowledge Discovery and Data Mining, AAAI Press / MIT Press, California, 249-271, 1996.

[31] Krantz, D.H., Luce, R.D., Suppes, P., and Tversky, A. Foundations of Measurement, Academic Press, New York, 1971.

[32] Lee, T.T. An information-theoretic analysis of relational databases - part I: data dependencies and information metric, IEEE Transactions on Software Engineering, 13, 1049-1061, 1987.

[33] Lin, T.Y., Yao, Y.Y., and Louie, E. Value added association rules, Proceedings of PAKDD, 328-333, 2002.

[34] Ling, C., Chen, T., Yang, Q., and Chen, J. Mining optimal actions for profitable CRM, Proceedings of ICDM, 767-770, 2002.

[35] Liu, B., Hsu, W., and Chen, S. Using general impressions to analyze discovered classification rules, Proceedings of KDD, 31-36, 1997.

[36] Liu, B., Hsu, W., and Ma, Y. Pruning and summarizing the discovered associations, Proceedings of KDD, 125-134, 1999. 
[37] Liu, B., Hsu, W., Han, H., and Xia, Y. Mining changes for real-life applications, Proceedings of Data Warehousing and Knowledge Discovery, 337-346, 2000.

[38] Liu, B., Hsu, W., and Ma, Y. Identifying non-actionable association rules, Proceedings of $K D D, 329-334,2001$.

[39] Liu, B., Hsu, W., and Ma, Y. Discovering the set of fundamental rule changes. Proceedings of KDD, 335-340, 2001.

[40] Liu, C., Zhong, N., and Ohsuga, S. " A multi-agent based architecture for distributed KDD process", Foundations of Intelligent Systems, Proceedings of 12th International Symposium (ISMIS 2000), 591-600, 2000.

[41] Luce, R.D. and Suppes, P. Preference, utility, and subjective probability, in: R.D. Luce, R.R. Bush, and E. Galanter, (Eds.), Handbook of Mathematical Psychology, John Wiley and Sons, Inc., New York, 249-410, 1965.

[42] Machie, J.L. Ethics: Inventing Right and Wrong, Penguin Books Ltd., Harmondsworth, 1977.

[43] Major, J. and Mangano, J. Selecting among rules induced from a hurricane database, The Journal of Intelligent Information Systems, 4, 1995.

[44] Mannila, H. Methods and problems in data mining, Proceedings of Database Theory, 41-55, 1997. 
[45] Mari, D.D. and Kotz, S. Correlation and Dependence, Imperial College Press, London, 2001.

[46] McClave, J.T. and Dietrich, F.H. Statistics, Sixth Edition, Macmillan College Publishing Company, Inc., Now York, 1994.

[47] Neumann, J. and Morgenstern, O. Theory of Games and Economic Behavior, Princeton University Press, 1944, 1947, 1953.

[48] Patton, M.Q. Practical Evaluation, Sage Publications, Newbury Park, 1982.

[49] Pawlak, Z. Rough Sets: Theoretical Aspects of Reasoning about Data, Kluwer Academic Publishers, Dordrecht, 1991.

[50] Pfanzagl, J. Theory of Measurement, John Wiley \& Sons, New York, 1968.

[51] Piatetsky-Shapiro, G. Discovery, analysis, and presentation of strong rules, in: G. Piatetsky-Shapiro, and W.J. Frawley (Eds.), Knowledge Discovery in Databases, AAAI/MIT Press, 229-238, 1991.

[52] Piatetsky-Shapiro, G. and Matheus, C.J. The interestingness of deviations, Proceedings of AAAI Workshop on Knowledge Discovery in Databases, 25-36, 1994.

[53] Plewis, I. Analysing Change: Measurement and Explanation Using Longitudinal Data, John Wiley \& Sons, New York, 1985. 
[54] Ras, Z. and Wieczorkowska, A. Action rules: how to increase profit of a company, Proceedings of PKDD, 587-592, 2000.

[55] Ras, Z. and Gupta, S. Global action rules in distributed knowledge systems, in: L. Czaja, H.D. Burkhard, and P. Starke (Eds.), Journal of Fundamenta Informaticae, 51, 175-184, 2002.

[56] Reith, D. Evaluation, a function of practice, in: J. Lishman (Ed.), Evaluation, Second Edition, Kingsley Publishers, London, 23-39, 1988.

[57] Roberts, F. Measurement Theory, Addison Wesley, Massachusetts, 1979.

[58] Sai, Y., Yao, Y.Y., and Zhong, N. Data analysis and mining in ordered information tables, Proceedings of ICDM, 497-504, 2001.

[59] Shafer, G. A Mathematical Theory of Evidence, Princeton University Press, 1976.

[60] Savage, L.J. The Foundations of Statistics, Dover, New York, 1972.

[61] Silberschatz, A. and Tuzhilin, A. On subjective measures of interestingness in knowledge discovery, Proceedings of KDD, 275-281, 1995.

[62] Silberschatz, A. and Tuzhilin, A. What makes patterns interesting in knowledge discovery systems, IEEE Transactions on Knowledge and Data Engineering, 8, 970-974, 1996. 
[63] Silverstein, C., Brin, S., and Motwani, R. Beyond market baskets: generalizing association rules to dependence rules. Data Mining and Knowledge Discovery, 2, 39-68, 1998.

[64] Simoudis, E. Reality check for data mining, IEEE Expert, 11, 26-33, 1996.

[65] Smyth, P. and Goodman, R. An information theoretic approach to rule induction from databases, IEEE Transactions on Knowledge and Data Engineering, 4, 301$316,1992$.

[66] Suchman, E.A. Evaluation Research, Russell Sage Foundation, USA, 1967.

[67] Tan, P.N. and Kumar, V. Interestingness measures for association patterns : a perspective. Technical Report TR00-036, University of Minnesota, 2000.

[68] Tan, P.N., Kumar, V., and Srivastava, J., Selecting the right interestingness measure for association patterns, Proceedings of $K D D, 2002$.

[69] Wang, K. and He, Y. User-defined association mining, Proceedings of PAKDD, 387-399, 2001.

[70] Wang, K., Zhou, S., and Han, J. Profit mining: from patterns to actions, Proceedings of EDBT, 70-87, 2002.

[71] Wang, K., Zhou, S., Fu, A., and Yu, J. Mining changes of classification by correspondence tracing, Proceedings of SDM, 2003. 
[72] Wille, R. Concept lattices and concept knowledge systems, Computers Mathematics with Applications, 23, 493-515, 1992.

[73] Wille, R. Restructuring lattice theory: an approach based on hierarchies of concepts, in: Ivan Rival (Ed.), Ordered sets, Reidel, Dordecht-Boston, 445-470, 1982.

[74] Wong, S.K.M., Yao, Y.Y., Bollmann, P., and Bürger, H.C. Axiomatization of qualitative belief structure, IEEE Transaction on Systems, Man, and Cybernetics, 21, 726-734, 1991.

[75] Wong, S.K.M. and Yao, Y.Y. Query formulation in linear retrieval models, Journal of The American Society for Information Science, 41, 334-341, 1990.

[76] Yang, J., Wang, W., and Yu, P.S. Infominer: mining surprising periodic patterns. Proceedings of KDD, 395-400, 2001.

[77] Yang, J., Wang, W., and Yu, P.S. InfoMiner+: Mining partial periodic patterns with gap penalties. Proceedings of ICDM, 725-728, 2002.

[78] Yao, Y.Y. Measuring retrieval performance based on user preference of documents, Journal of the American Society for Information Science, 46, 133-145, 1995. 
[79] Yao, Y.Y. A step towards the foundations of data mining, Proceedings of Data Mining and Knowledge Discovery: Theory, Tools, and Technology V, The International Society for Optical Engineering, 254-263, 2003.

[80] Yao, Y.Y. Information-theoretic measures for knowledge discovery and data mining, in: Karmeshu (Ed.), Entropy Measures, Maximum Entropy and Emerging Applications, Springer, Berlin, 115-136, 2003.

[81] Yao, Y.Y. On modeling data mining with granular computing. Proceedings of COMPSAC, 638-643, 2001.

[82] Yao, Y.Y., Chen, Y.H. and Yang X.D. Measurement-theoretic foundation for rules interestingness, ICDM 2003 Workshop on Foundations and New Directions in Data Mining, 221-227, 2003.

[83] Yao, Y.Y. and Liau, C.J. A generalized decision logic language for granular computing, FUZZ-IEEE on Computational Intelligence, 1092-1097, 2002.

[84] Yao, Y.Y. and Sai, Y. Mining ordering rules using rough set theory, Bulletin of International Rough Set Society, 5, 99-106, 2001.

[85] Yao, Y.Y., Wong, M.S.K., and Butz, C.J. On information-theoretic measures of attribute importance, Proceedings of PAKDD, 133-137, 1999. 
[86] Yao, Y.Y., Zhao, Y., and Maguire, R.B. Explanation-oriented association mining using a combination of unsupervised and supervised learning algorithms, Proceedings of Canadian Conference on AI, 527-532, 2003.

[87] Yao, Y.Y. and Zhong, N. An analysis of quantitative measures associated with rules, Proceedings of PAKDD, 479-488, 1999.

[88] Yao, Y.Y., Zhong, N. and Ohshima, M. An analysis of Peculiarity oriented multidatabase mining, IEEE Transactions on Knowledge and Data Engineering, 15, 952-960, 2003.

[89] Watanabe, S. Pattern recognition as a quest for minimum entropy, Pattern Recognition, 13, 381-387, 1981.

[90] Zhong, N., Liu, C., and Ohsuga, S. "Dynamically organizing KDD processes", Journal of Pattern Recognition and Artificial Intelligence, 15, 451-473, 2001. 